\title{
A Selection Model for Motion Processing in Area MT of Primates
}

\author{
Steven J. Nowlan ${ }^{a}$ and Terrence J. Sejnowski \\ Howard Hughes Medical Institute, Salk Institute for Biological Studies, San Diego, California 92186-5800 and Department \\ of Biology, University of California at San Diego, La Jolla, California 92093
}

\begin{abstract}
A computational model for motion processing in area MT is presented that is based on the observed response properties of cortical neurons and is consistent with the visual perception of partially occluded and transparent moving stimuli. In contrast to models of motion processing that assume spatial continuity and fail to compute the correct velocity for these visual stimuli, our model produces a distributed segmentation of the image into disjoint patches that represent distinct objects moving with common velocities. A key element in the model is the selection of regions of the visual field where the velocity estimates are most reliable. The processing units in the motion model that perform the selection have nonclassical receptive fields similar to those observed in area MT (Allman et al., 1985). The psychophysical responses of the model to coherently moving random dots and transparent plaid gratings are similar to those observed in primates.
\end{abstract}

[Key words: visual cortex, network model, motion processing, area MT, motion transparency, nonclassical receptive fields]

Our ability to process rapidly the complex and confusing motion signals in visual scenes is impressive. We can estimate motion direction (Levinson and Sekuler, 1976) and speed (McKee, 1981; McKee and Nakayama, 1984; McKee et al., 1986) with high precision and visually track one out of multiple moving objects, despite the presence of opaque or transparent occluding surfaces, varying illumination, and moving backgrounds. In addition to psychophysical studies, the organization of primate vision systems provide important constraints on how motion is computed. In this article we explorc a computational model of motion processing that is broadly consistent with our current knowledge of the primate visual system.

Cells with reliable selectivity for direction of motion are found in area V1 of primate visual cortex (Nakayama, 1985; Maunsell and Newsome, 1987); these cells do not detect true velocity, but, instead, are tuned to a limited range of spatiotemporal frequencies (Maunsell and Newsome, 1987, Andersen and Siegel, 1989). In addition, these cells exhibit marked orientation selectivity and spatially restricted receptive fields so that they

\footnotetext{
Received Oct. 1, 1993; revised July 22, 1994; accepted July 27, 1994.

We thank Drs. Gene Stoner, Thomas Albright, Paul Viola, and Stephen G. Lisberger for helpful discussions on motion processing in area MT, and Drs. Ning Qian, Norberto Grzywacz, Paul Viola, J. Anthony Movshon, and Thomas Albright for comments on this manuscript. This research was supported by the Howard Hughes Medical Institute and the Office of Naval Research.

Correspondence should be addressed to Terrence J. Sejnowski, CNL, The Salk Institute, P.O. Box 85800, San Diego, CA 92186-5800.

apresent address: Synaptics Inc., 2698 Orchard Parkway, San Jose, CA 95134 Copyright (C) 1995 Society for Neuroscience $0270-6474 / 95 / 151195-20 \$ 05.00 / 0$
}

can only report the component of motion perpendicular to an oriented edge. This perpendicular component cannot fully disambiguate the direction and speed of motion, and this failure is sometimes called the "aperture problem." In order to overcome these limitations and compute true local velocity measurements, it is necessary to integrate motion responses from cells with a variety of directions and spatiotemporal frequency tunings over a wider area of the visual field (Heeger, 1987; Grzywacz and Yuille, 1990).

Lesion studies provide evidence that area $\mathrm{MT}$, which receives a direct projection from area $\mathrm{V} 1$, plays a crucial role in speed and, to a somewhat lesser extent, direction perception of moving stimuli (Newsome et al., 1985; Newsome and Pare, 1988; Andersen and Siegel, 1989). Area MT is the first visual area where neurons respond selectively to velocity over a wide range of spatial frequencies (Albright, 1984; Maunsell and Newsome, 1987; Rodman and Albright, 1989; Albright, 1992). In particular, a class of cells in area MT, the "pattern cells" of Movshon et al. (1985), respond to the direction of overall motion of plaid patterns composed of two differently oriented sinusoidal gratings, rather than to the direction of the individual components. Neurons with this type of motion response, which are able to solve the aperture problem, are common in area MT but rare in area V1 (Ferrera and Maunsell, 1991).

Psychophysical studies have suggested that the perceived velocity of such plaid patterns is generally close to the velocity that is uniquely consistent with the constraints imposed by the individual component motions, as shown in Figure 1 (Adelson and Movshon, 1982; Welch, 1989), although other possibilities have been suggested (Wilson et al., 1992; Rubin and Hochstein, 1993). In this article, we are concerned with the properties of such pattern motion cells and their responses to two other types of motion stimuli that have previously been used in psychophysical and physiological experiments: randomly moving dots (Newsome and Pare, 1988) and transparent plaids (Stoner et al., 1990).

The computational model studied here shows how local velocity responses in area MT could be combined to represent the velocity of objects in visual scenes, even when there are occluding and transparent objects present (Nowlan and Sejnowski, 1994). The model departs from previous methods for estimating local velocity that attempt to estimate the local velocity at all image locations, called the optical flow field (Horn and Schunk, 1981; Heeger, 1987, 1992; Grzywacz and Yuille, 1990). Most of these approaches to motion processing assume spatial continuity and spatially average spatial and temporal frequencies estimates over local neighborhoods. In contrast, our model cvaluates the reliability of local velocity estimates and selects a coarse, discontinuous representation of the motion of objects in the visual scene by combining only the most valid subsets of 


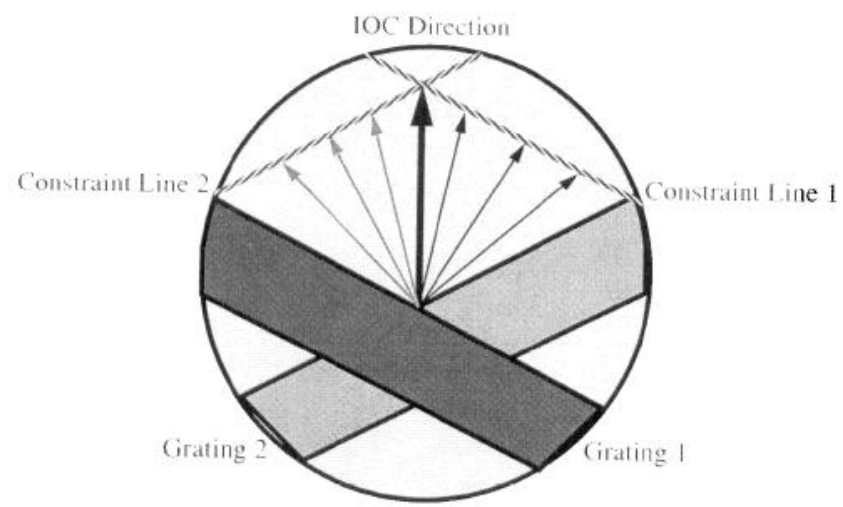

Figure 1. Pattern motion for two gratings. The light and dark bars correspond to two different gratings moving within the restricted receptive field. The arrows correspond to possible velocities of the bars. For each bar, many velocities are consistent with the local motion of the bar; for the dark bar, any arrow whose tip touches the line with diagonal downward stripes is consistent with the motion of that bar This represents the velocity constraint line for the dark bar. The line with diagonal upward stripes is the velocity constraint line for the light bar. There is only a single velocity that is simultaneously consistent with the motion of both bars. This velocity, indicated by the heaviest arrow at the intersection of the two constraint lines, is called the intersection of constraints (IOC) velocity.

local velocity measurements. The local velocity estimates and the reliability estimates are computed in parallel by fast, feedforward processing in simple networks.

We used an optimization procedure to construct the model because, at present, we know of no other way to solve the motion problem as we have defined it. We do not mean to imply that the brain must use the same optimization procedure. On the contrary, we believe that the nervous system uses a combination of genetically determined and adaptive mechanisms to produce a computational system for interpreting the range of moving objects and configurations that commonly occur in the world. Our strategy was to start with a model that matched the basic architecture of the visual system and to make computational assumptions that matched known psychophysical constraints. Optimization was then used to fill in the missing parameters. Following optimization, the responses of units in the model were found to match the responses of real neurons to the same visual stimuli, and the performance of the model matched the psychophysical performance of primates on a number of visual stimuli including ones that were not in the optimized set of inputs (see Nowlan and Sejnowski, 1994, for additional examples).

In this article, we compare in detail the properties of the units in our model with the properties of neurons found in area MT of the primate visual cortex. The essentially parallel and feedforward nature of the model has several advantages in speed and simplicity over more complex models that depend on extensive lateral processing within an area (Horn and Schunk, 1981; Zucker et al., 1981; Kienker et al., 1986; Darrell and Pentland, 1991). In our model, lateral interconnections are used primarily for competition and normalization, which can be implemented in a variety of ways with mechanisms that are known to exist in the cortex (see Discussion). The model yields accurate velocity estimates from synthetic images containing multiple moving targets of varying size, luminance, and spatial frequency profile, including occlusion and transparency.

The architecture of the model is briefly described in Materials and Methods. Details of the mathematical derivation can be found elsewhere (Nowlan and Sejnowski, 1994). In the Results, several examples are given of how the model responded to simple moving stimuli; the response characteristics of units within different stages of the model are related to physiological properties of units in the primate visual system; and the model is tested with several visual stimuli that have been used in psychophysical experiments to compare its performance with human motion perception. Finally, some of the general and specific implications of the model are presented in the Discussion. An earlier one-dimensional version of the model has already appeared (Nowlan and Sejnowski, 1993).

\section{Materials and Methods}

The inputs to our network model of area MT are models of the responses of neurons in area V1 that project to MT. We have not attempted to account for how these directional responses are actually synthesized by neurons in V1; nor have we attempted to replicate in detail the biophysical interactions that occur between neurons in area MT. The processing units in the network are only meant to capture the responses that are observed at the level of the average firing rates of neurons. The model provides a framework for studying how the signals carried by MT neurons could be used to estimate the velocity of moving objects in the presence of occlusion. The computational operations in the model are relatively simple ones, such as summation and normalization, which can be implemented by real neurons in a variety of ways (see Discussion).

Soft-maximum is an example of a basic computational operation that occurs at several stages of processing in our model. The purpose of softmaximization is to enhance the firing rate of the unit that has the highest firing rate and to normalize all of the responses in the population so that the total activity is a constant, regardless of their initial firing rates. This can be accomplished mathematically by the function

$$
R_{k}(x, y)=e^{u\left(R_{k}^{\prime}(x, y)\right.} / \sum_{x^{\prime}, y^{\prime}} e^{\alpha R t\left(x^{\prime}, y^{\prime}\right)},
$$

where $R_{k}^{\prime}$ are the initial firing rates and $R_{k}$ are the normalized firing rates, $(x, y)$ are spatial locations that index the population of units to be normalized, and $\alpha$ is a constant that determines the degree of separation of the highest firing rate from all the others. The first step is to amplify all of the firing rates by exponentiation, the second step is to compute the sum of all of these amplified responses, and the third step is to normalize the responses by dividing by their sum. The net effect of all three operations is to emphasize the differences between the units with the highest levels of activity and all the others in the population. As the amplification constant is increased, the differences are further enhanced until, in the limit as the constant becomes very large, one unit fires at its maximum rate and all the rest are reduced to zero. This limit is called winner take all. A soft-maximum operation could be implemented in cortex by amplification of the inputs through recurrent excitation between local units in the population followed by mutual inhibition to normalize the responses (Hadeler, 1974; Feldman and Ballard, 1982; Carandini and Heeger, 1994), but other circuits can also perform the task that are faster and more reliable (Yuille and Grzywacz, 1989).

The general design of the model is a cascade of stages, each consisting of an array of processing units that are locally connected and arranged in a roughly retinotopically organized map (Fig. 2). Within each stage there are a number of "layers" or "channels," each covering the visual field. For example, for each of several directions of movement there is an array of neurons that together provide an overlapping map of the visual field, in the same way that orientation is represented in area V1. Processing is divided into four main stages that are summarized here and described in greater detail in Nowlan and Sejnowski (1994). The retinal and motion-energy stages are accomplished with fixed filters, each of which can be computed by a feedforward network of converging and diverging connections. The subsequent stages of processing are adaptive filters in the sense that the connection strengths in the model were not predetermined but were, instead, found by an optimization procedure. All of the results presented here were obtained from one network with fixed connection strengths produced by this procedure.

The model was constrained by anatomical and physiological consid- 


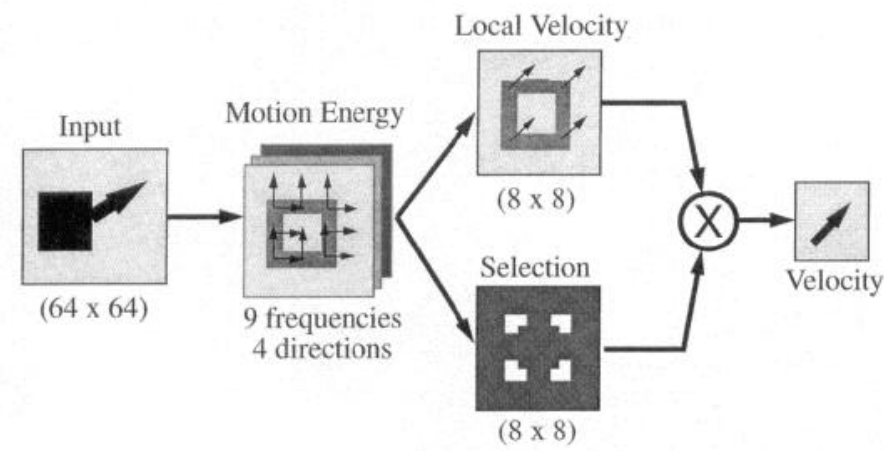

Stage 1

Stage 2

Stage 3

Figure 2. Schematic diagram of motion processing model. Processing proceeds from left to right in the model, starting with the retinal input and proceeding through three feedforward stages of processing. The local velocity and selection stages operate in parallel. Shading within the boxes indicates different levels of activity at each stage. Arrows within motion-energy and local velocity stages indicate local directions of motion responding units. The responses shown are intended to be indicative but do not represent actual responses from the model.

erations as well as by computational goals. The fixed stages of the model and the computational principle used in the local velocity and selection stages are described first. Although the architectures of the local velocity and selection stages were fixed, the properties of units in them were determined by a procedure used to optimize the overall performance of the model to occluding and transparent moving stimuli. Finally, we present the detailed response properties of the units in these stages.

Retinal processing. The $64 \times 64$ pixel input array is roughly equivalent to an array of photoreceptors that represents the intensity at each pixel location by one of 256 gray levels. A motion stimulus consists of a sequence of images that are processed first through the retinal stage and subsequently through spatiotemporal filters, as described in the next section. The retinal stage of processing contrast enhances each image with a difference-of-Gaussian filter at each location of the array. (The excitatory Gaussian has a standard deviation of three pixels and the inhibitory surround has a standard deviation of seven pixels.) This is similar to the processing that occurs in retinal on-center ganglion cells. It removes the constant component of intensity across the image, smooths the noise, and enhances the edges.

Motion-energy filters. In the first stage of motion processing, a distributed representation of motion is extracted and used as the inputs to the model of area MT. We use the motion-energy model of Adelson and Bergen (1985), which consists of arrays of spatiotemporal filters, each tuned to a particular direction of motion and sensitive to a particular combination of spatial and temporal frequency. Altogether, there are 36 channels of motion-energy filters tuned to four directions and nine combinations of spatial and temporal frequency. Each filter receives input from a $16 \times 16$ patch of the retinal output. These filters are broad and overlapping in their selectivities and their velocity tuning depends on the spatial characteristics of the moving pattern. They are constructed by nonlinearly combining oriented linear spatiotemporal filters to produce an output that is insensitive to the spatial phase of the visual stimulus (the response is invariant to exchange of light and dark regions). The properties of motion-energy filters resemble those of directionally tuned complex cells in the visual cortex of cats and monkeys (Fig. 3) (Maunsell and Newsome, 1987; Emerson et al., 1992; Qian and Andersen, 1994).

The motion-energy filters form a $49 \times 49$ array, each location of which contains the outputs of 36 filters. This is analogous to a cortical motion hypercolumn that implicitly contains all the information needed to extract the velocities of moving objects. However, before passing this information on to subsequent stages of processing, the outputs of these filters are normalized by a soft-maximum operation (see above) so that the summed activity over all $\mathbf{3 6}$ motion-energy filters in a hypercolumn is a constant. This is consistent with the responses observed in cortical cells as spatial contrast was varied (Albrecht and Geisler, 1991; Heeger, 1992). The purpose of normalization is to represent the relative rather

\section{Quadrature pair}

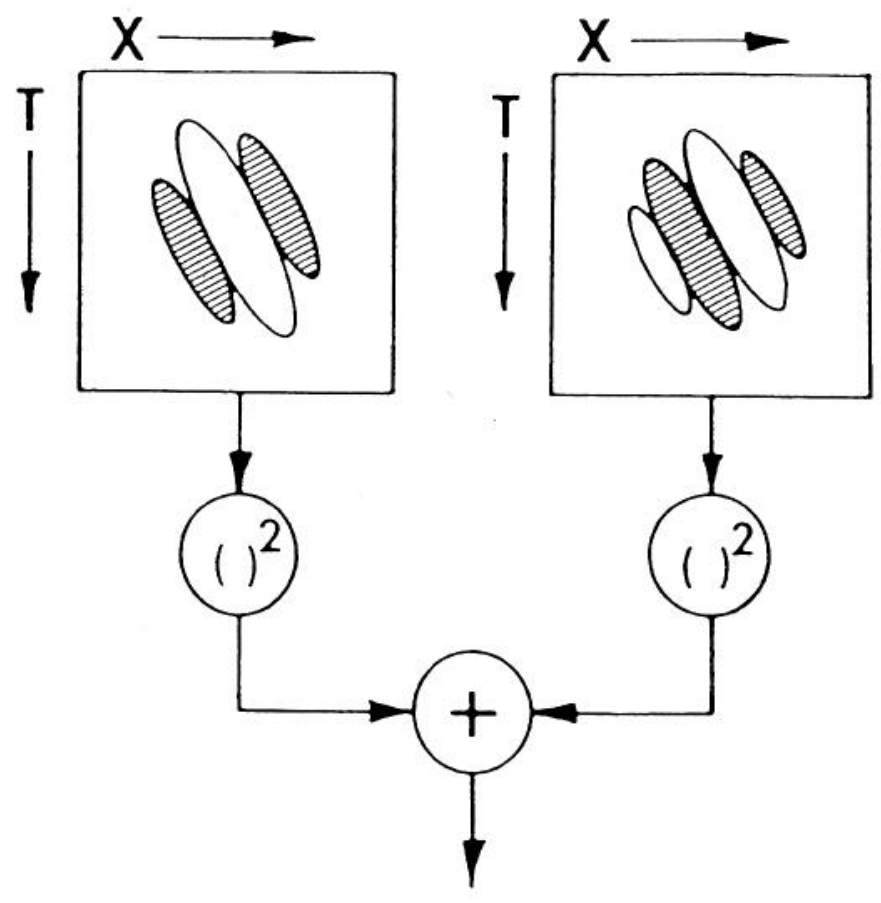

\section{Motion energy}

Figure 3. Nonlinear motion-energy filter used in the motion model to represent the processing in area $\mathrm{V} 1$. The two oriented spatial-temporal filters, shown at the top, have responses that are $90^{\circ}$ out of phase (a quadrature pair). The output of the two filters are first squared and then summed to produce the final output of the filter. This motion-energy filter gives a strictly positive, phase invariant response that is maximal for a particular spatial and temporal frequency combination. (From Adelson and Bergen, 1985.)

than absolute values for contrast and motion properties, taking maximum advantage of the limited dynamical range of the output firing rates of neurons. The next stage of motion processing has two processing streams that proceed in parallel, each taking as their inputs the normalized motion-energy outputs.

The selective integration principle. The principle of selective integration provides a way to effectively compute quantities that depend on noisy data widely distributed among many inputs. This approach is applicable when a number of different estimators are available for that quantity, each of which works well under a limited set of conditions. A good estimate over a large range of conditions can then be achieved by combining estimates that work well under each set of conditions, and ignoring estimates that are poor under those conditions. We can accomplish this by using as our overall estimate a weighted average of the estimates:

$$
V(x)=\sum_{k} S_{k}(x) V_{k}(x)
$$

where $V_{k}(x)$ is the estimate from estimator $k$ based on the current conditions $x$ and $S_{k}(x)$ is large if estimator $k$ works well under the current conditions and small otherwise. We must ensure that $\Sigma_{k} S_{k}(k)=1$ for all $x$ so that we have a true weighted average under all circumstances.

This principle can be applied if two problems are solved: first, a good set of estimators is needed that span the conditions of interest, and second, there must be means for predicting accurately which estimators to use under each particular set of conditions. Equation 2 is symmetric in the estimators $V_{k}$ and the weights $S_{k}$ and so it cannot be used directly to find good sets of $V_{k}$ and $S_{k}$. Instead, we use an optimization procedure that is based on how good an estimate each $V_{j}$ is relative to all of the other $V_{k}$. 


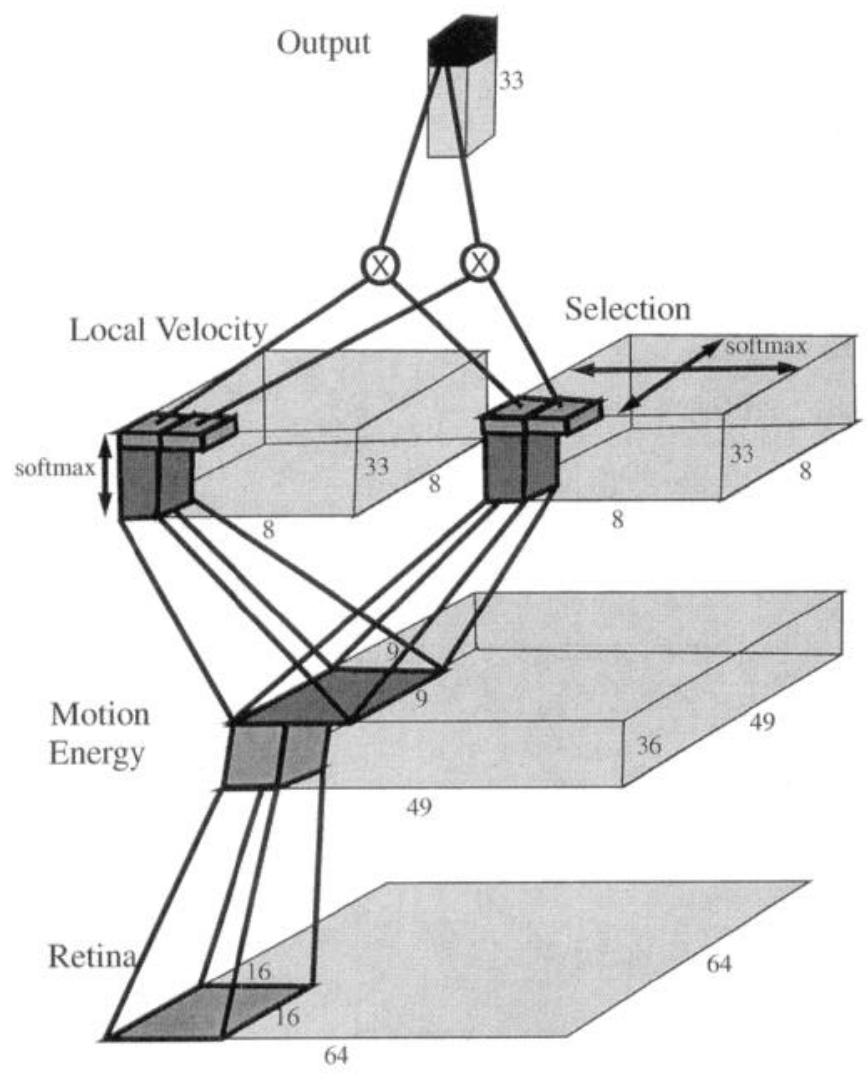

Figure 4. Detailed architecture of the model showing bottom-up feedforward convergence of motion information. The retina is a 64 by 64 pixel array. Each motion-energy column contained 36 units that received inputs from $16 \times 16$ regions of the retina. Local velocity and selection columns contained 33 units. For each local velocity column there was a corresponding selection column that received input from the same $9 \times 9$ array of motion-energy columns. Since adjacent motionenergy columns only have partially overlapping receptive fields, the effective receptive field size of the local velocity and selection columns were 81 times larger than the receptive field size of the motion-energy units. Competition (soft-maximum) occurs between units within each local velocity column and between units with the same velocity preference across selection columns, as shown by the arrows. The values of the 33 units in the single output column were determined by multiplying the outputs of corresponding pairs of local velocity and selection units and summing across a spatial array of these units. The output is the final estimate of the velocities of all the objects moving within the 64 $\times 64$ pixel array. The velocity of more than one object may be represented in the output column at the same time as long as the velocities are not too similar. There are a total of 8.8 million weights in the network, but because of translational symmetries only 138,600 of them are independent. In a complete model of area MT there would be a spatial array of output columns.

If $D\left(V, V_{k}\right)$ is a measure of the distance between the true value we wish to estimate and a particular estimate of that value, we assign a relevance to each $V_{k}$ :

$$
R_{k}=\frac{S_{k} D\left(V, V_{k}\right)}{\sum_{k} S_{k} D\left(V, V_{k}\right)}
$$

Initially we have all of the $S_{k}$ equal, so $R_{k}$ is simply the distance for this particular estimate, divided by the average distance of all of the estimates. Good estimates will have large values of $R_{k}$ and poor estimates will have small values for $R_{k}$. The estimates $V_{k}$ are optimized by minimizing an error proportional to $R_{k} D\left(V, V_{k}\right)$ across a set of training examples where the true $V$ is known. $V_{k}$ is assigned a large fraction of the error when $R_{k}$ is large and a small amount of error when $R_{k}$ is small; the error is then used to improve the estimator which, in this way, becomes a better estimator over a limited set of conditions. The weights $S_{k}$ are optimized by minimizing an error proportional to $R_{k}-S_{k}$. Each $S_{k}$ becomes a good approximation to $R_{k}$, which is a good measure of how much weight should be assigned to $V_{k}$ under current conditions. The details of this optimization procedure and the underlying statistical model are described elsewhere (Nowlan, 1990; Jacobs et al., 1991; Nowlan and Sejnowski, 1994).

The local velocity and selection stages of our model are based on applying the principle of selective integration to the problem of estimating velocity in a visual scene. Units in the local velocity network compute estimates of velocity based only on information from a limited region of the visual scene. Not all of these velocity estimates will be accurate. Regions that contain occlusion, transparency, or lack oriented contours may provide little or no useful velocity information and should be ignored as long as other regions of the scene contain more reliable velocity information. The purpose of the selection network is to weight estimates from each region of the image according to how reliable those estimates are.

Model constraints. The properties of units in the selection and local velocity pathways were determined by the selective integration optimization procedure rather than being set a priori. The properties of these units also depended on the architecture of the selection and integration networks. Constraints were imposed by the choice of how to represent velocity in the model, the input received by the selection and local velocity units, and the organization of the soft-maximum normalization in the local velocity and selection networks.

Our model used a distributed representation of velocity, with 33 different velocity channels. There were eight different directions corresponding to equally spaced compass points and four different velocities, 32 different combinations, plus one more unit that represents zero velocity. For each of these velocity channels, we computed a separate estimate of whether that velocity was present in the visual scene:

$$
v_{k}(t)=\sum_{x, y} I_{k}(x, y, t) S_{k}(x, y, t),
$$

where $v_{k}(t)$ is the global evidence at time $t$ for a visual target moving at a particular velocity $k, I_{k}(x, y, z)$ is the local evidence for velocity $k$ computed by the local velocity pathway from region $(x, y)$ at time $t$, and $S_{k}(x, y, t)$ is the weight assigned by the selection pathway to that region. $v_{k}(t)$ represents the activity of one of the 33 velocity units in the pool of output units (Fig. 4). There was a single population of output units in the model, so that the output stage has access to information across the entire visual field. This lack of spatial resolution at the output stage is one of the major simplifications of the current model.

The choice of output representation immediately constrains the organization of the local velocity and selection networks. The selective integration principle requires that there is a one-to-one correspondence between the representation of velocity at the output stage and the representation in the local velocity network. The local velocity network is thus organized as an array of $8 \times 8$ locations each containing 33 velocity units. The smaller size of the spatial array relative to the motion-energy stage reflects the convergence of inputs and concomitant increase in receptive field size, as observed in the visual pathway from area V1 to area MT. The selective integration principle also requires a one-to-one correspondence between local velocity units and selection units so that the selection network must also be organized as an array of $8 \times 8$ locations with 33 selection units in each location.

The choices in the design of the retinal and motion-energy stages of our model constrain the input to the local velocity and selection units. The input for each local velocity and selection unit was a field of $9 \times$ 9 motion-energy units (see Fig. 4). The motion-energy layer had only four directions of motion represented, but eight directions were represented in the local velocity and selection networks. Selection and local velocity units received input from all motion-energy channels with directional tuning within $90^{\circ}$ of the directional tuning of the velocity or selection channel. Thus, some local velocity and selection units received input from motion energy units with a single directional tuning, while others received input from motion energy units with two different directional tunings.

The final source of constraint was imposed by the organization of normalization in the selection and integration networks. Units in both networks computed linear weighted summations of their inputs followed by a soft-maximum operation (Eq. 1), applied differently in the selection and local velocity networks. This introduced a nonlinearity into the responses of these units. 
For the selection units, the soft-maximum operation was applied separately to each of the selection channels. Equation 2 requires that the weights assigned to a particular estimate must sum to one; that is, the outputs of each of the 33 selection channels must be normalized spatially. This operation emphasized the responses in spatial locations with the most reliable information and suppressed those locations with the least reliable information.

The soft-maximum normalization in the local velocity network identified the most likely velocity estimate computed from the local motion energy measurements. For a single, rigidly moving object in the visual field with no occluding or transparent objects, relatively simple averaging schemes have been used to estimate the local velocity (Heeger 1987; Grzywacz and Yuille 1990; Ileeger 1992). In these approaches, a linear weighted summation was performed for each direction of motion and speed and the maximum taken across these channels. In our model, the maximum operation was accomplished with a soft-maximum operation over all 33 units in a spatial column, which allows more than one velocity estimate to survive.

Initialization and training. The local velocity and selection units were initialized with weights that had small random values, so that all of their responses were initially very weak. At this stage, the velocity units showed no consistent velocity tuning, nor did the selection units exhibit strong selective preferences. The properties of both types of units were determined indirectly, by optimizing the model to produce the correct velocity in the final output layer of units for a wide range of moving stimuli.

Since the responses of selection and velocity units were primarily determined during optimization, the statistics of the set of patterns used to train the model had a significant influence. The input patterns used to optimize the performance of the network were 500 "movies" of moving objects, such as the examples shown in Figures 6 and 7. The known velocity of each object was used to modify the weights in the appropriate local velocity network, and the weights in the selection networks were changed to identify the most reliable regions. The training set had examples of occlusion and multiple moving objects, including interactions between transparent objects that were based on a physical transparency model. The exposure to transparency and occlusion during training is critical to allowing the model to develop robust methods for dealing with the presence of both phenomena in real scenes. The training set did not contain examples of the plaid patterns or the random-dot pattern that were used to test the model, and these provided an important test of the ability of the model to generalize to novel stimuli.

Local velocity network. The optimized responses of the velocity units were broadly tuned around their best directions and velocities so that a given motion stimulus would produce a broadly distributed pattern of activity in these 33 units. The unit with the maximum response represented the most likely local velocity in that patch of the visual field. Rather than choose among the 33 units in the column the single unit with the maximum response, the model used a soft-maximum to enhance the largest responses (Eq. 1). A winner-take-all mechanism would lose valuable information, especially when the true velocity was between the velocities represented by particular units. Using a softmaximum also allowed more than one velocity to be represented in each pool. If the output of each unit is considered the evidence for a particular velocity in a particular region of the image, then the constraint that the sum of activity in the pool of 33 local velocity neurons must equal one is equivalent to assuming that the total evidence across all velocities must sum to one.

Selection network. When the trained selection units were probed with moving objects, their largest responses tended to occur at regions of the image where there was a discontinuity in the distribution of motionenergy inputs. However, because they were optimized to respond primarily to the patterns of discontinuities that characterize regions of reliable support for object motion, the algorithm that they implement is more specialized than a simple discontinuity detector. The selection units responded best to borders between different velocities only over a restricted range of orientations and speeds. As a result, they responded strongly only to motion discontinuities that were consistent with object motion with a particular speed and direction (Fig. 5). The modcl also selected subsets of spatial locations over which to integrate the local velocity field, which could be disjoint. This allowed the model to account for interpenetrating motion fields for which the assumption of spatial continuity of the velocity field was invalid.

In the next section, the performance of the model on examples of inputs similar to those used to construct the model is described. The a)

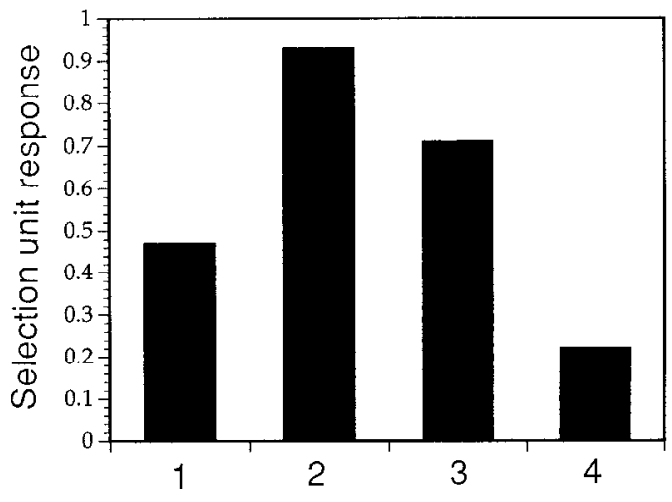

1

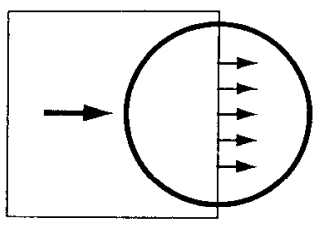

3
2

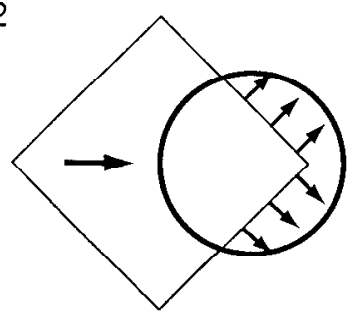

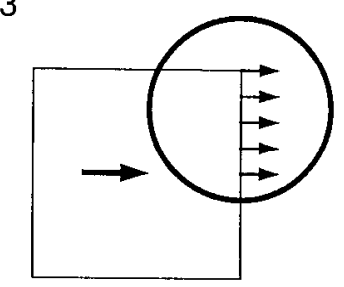

4

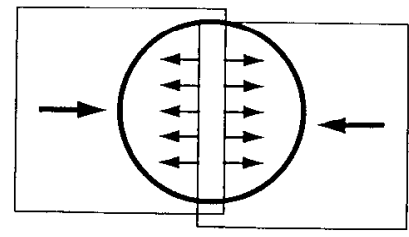

Figure 5. Responses of a selection unit to motion discontinuities. $a$, The bar graph shows the strength of the response of a selection unit tuned to rightward motion to four different distributions of motionenergy responses within the selection unit's receptive field. $b$, Schematic diagram of four motion-energy distributions. In the first three examples, the square is moving in the direction indicated by the large arrow against a stationary background. In the last example, two squares, one semitransparent, move against a stationary background. The circle indicates the receptive field of the selection unit and the small arrows indicatc the direction of local motion reported by the motion-energy units within this receptive field. In example 1, the receptive field is centered over an edge moving to the right and sees a uniform distribution of rightward motion-energy responses producing a moderate response $(0.48)$ from the unit. In contrast, the response to a similar motion in example 3 is much stronger $(0.74)$ since, in this case; the receptive field includes a corner region that contains a discontinuity between a region of rightward motion-energy response and a region containing no motion-energy response. The strongest response from this selection unit $(0.93)$ is seen in example 2, where the receptive field encloses a discontinuity between two orthogonal sets of local motion-energy measurements. Finally, in example 4 the local motion-energy responses corresponding to two opposed motions (generated in this case by one transparent object moving in front of a second moving object) suppresses the response of a selection unit. This last example shows that the selection unit is directionally tuned. The response of a single selection unit is shown here in isolation. The overall response of a selection unit is determined by its own local response in competition with the responses of similarly tuned selection units in other image regions.

results are instructive in helping to explain the operation of the model The properties of the local velocity and selection units were obtained by presenting the model with a variety of standard motion stimuli, such as oriented gratings. Finally, we tested the model on novel visual stimuli 
a)

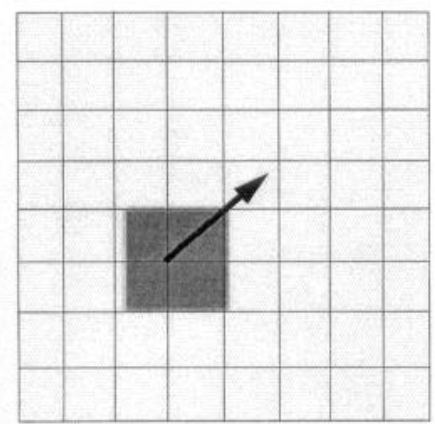

b)

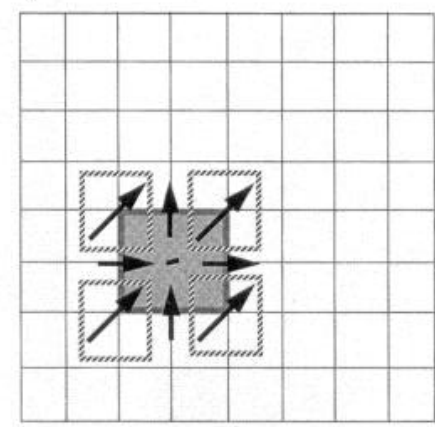

Figure 6. Illustration of local velocity and selection responses to a single moving object. $a$, The input to the model is a square object of uniform intensity moving at 0.5 pixels per frame at a heading of $45^{\circ}$, as indicated by the heavy arrow. Intersections of grid lines correspond to locations of centers of local velocity and selection receptive fields. $b$, Representation of the outputs of the local velocity and selection stages of the model for this stimulus. The arrows at grid points represent the output of the local velocity pool at that receptive field location. Regions enclosed in dashed lines correspond to local velocity measurements selected for integration at the output stage.

used in psychophysical experiments and compared the predictions of the model with human performance.

\section{Results}

\section{Performance on moving objects}

The local velocity estimation and selection pathways work in parallel to segment an image coarsely into coherent motion regions and estimate the velocity of these regions. The examples are of image sequences containing one or two simple objects moving against a stationary background. More complex examples are presented in later sections. For each example, we show the input to the model, a representation of the local velocity estimates and how these estimates are grouped by the selection pathway. An intuitive grasp of the how the system functions helps in interpreting the formal characterization of the system presented in the next section.

A square "object" of uniform intensity moving northeast is shown in Figure $6 a$. The dashed grid superimposed on this stimulus indicates the coarser representation of this space at the local velocity and selection stages of the model. Figure $6 b$ shows the local velocity estimates for this input. (All of the figures in this section may be thought of as showing a snapshot of the input and corresponding activity at higher stages of the model at one instant of time. The activity of the later stages would not be based on just this one input frame but also on several preceding frames.) Each velocity estimate is represented by a small arrow, indicating the magnitude and direction of the estimate. In addition, dashed lines enclose the estimates that make the main contribution to the calculation of overall velocity in the scene. The contribution of a velocity estimate to the overall velocity is the product of the activity of a particular velocity unit and the corresponding selection unit, and both of these must be large for an estimate to contribute to the overall output of the model. Since, in this scene, there is a single global motion, only the support for this motion is illustrated.

This example reveals how our model handles some important aspects of motion processing. The central region of the object contained no contrast variations, so even though this region was moving along with the rest of the object, velocity estimates centered in this region report no velocity. The velocity estimates near the center of the object's edges were based on contrast edges of a single orientation. As a result, these estimates could only represent the component of motion orthogonal to these edges. The only local velocity estimates that reflected the true motion of the object were those from regions near the four corners of the object. In these regions, the presence of edges at two orientations was sufficient to disambiguate the true velocity.

The corner regions produced strong selection unit activity due to the presence of motion discontinuities at the convergence of two edges. This distribution of motion excited selection units for upward, rightward, and the northeast diagonal, with the diagonal selection units most strongly activated. The local velocity pools in these corner regions also had their activity concentrated in the unit corresponding to motion along the northeast diagonal. In order to understand the output produced by the model, the interaction between the local velocity responses and the selection responses for the three candidate motions must be compared. For motion along the northeast diagonal, the selection unit activities were concentrated in the four corner regions exclusively, and in these regions the local velocity pool activities were also concentrated on the northeast diagonal motion. As a result, the product of the selection and the velocity activities in these corner regions made a strong contribution to the northeast diagonal global velocity unit. For either rightward or upward motion, the selection activities were also greatest in the corner regions. However, in these regions, activity in the local velocity pool was concentrated in the units representing northeast diagonal motion so the local velocity activity for right and up was very weak. Conversely, for the vertical and horizontal object edges between the corners, the activity in the local velocity pools was concentrated in the units indicating upward or rightward motion, but the selection activity was quite weak since it was concentrated in the corners. As a result, the overall contribution to upward or rightward motion was very small.

Averaging the weighted local velocity estimates produced a good overall estimate of the velocity of the object. The activity of the selection units segmented from the scene regions of coherent motion; note, however, that these regions were disjoint and do not correspond to the entire object. The segmentation would be the same if the original scene had contained only the corners of the object rather than the entire object. Some other process would be required to "fill in" the region corresponding to the object (just as a Kanisza triangle can be filled in based only on the presence of its corners). This other process could use texture, color, or intensity cues to fill in this surface.

The second example was composed of two objects moving in opposite directions (Fig. 7a). One object was striped horizontally and moved to the right with a speed that was half that of the second object, which was striped vertically and moved to the left. The local velocity estimates and regions contributing strongly to the global velocity are shown in Figure $7 b$. In this case, regions contributing to rightward motion are denoted with a light line, while those contributing to leftward motion are denoted with a dark line. (Recall that separate regions of support were computed for different candidate global velocities.) In this example, the leftward moving object was on top of the rightward moving object (i.e., closer to the observer), and was composed of a material that reflected but did not transmit light. As a result, in regions where the two objects overlapped, the second object occluded the first object totally and the intensity in these regions was the intensity of the second object. 
In Figure $7 b$ the local velocity estimates in the region of overlap of the two objects are marked with double arrow heads. In these regions, the activity in the local velocity pool was not concentrated in a single unit but was distributed bimodally with activity peaks corresponding to two opposing directions of motion. In this ambiguous region, the velocity unit activities for leftward and rightward motion were relatively weak due to the bimodal activity distribution. In addition, this opposed motion distribution did not strongly excite selection units for either leftward or rightward motion. As a result, this ambiguous region was not included in the region of support for the motion of either object.

Note also that for the rightward moving object, the stripes were oriented parallel to the direction of motion and regions near the center of these stripes experienced no contrast variation. Hence, these contrast borders failed to respond to the motion of the object and the major contribution to rightward global motion came only from the leading and trailing edges. For the leftward moving object, the contrast stripes were perpendicular to the direction of motion, providing strong motion signals over most of the region covered by the object. As a result, the regions of support for this object were correspondingly much larger.

These two examples illustrate some of the complexity of the interactions in the model. In the first example, velocity measurements near the center of object edges were not contained in the final regions of support for the object motion. This was because the corner regions of the object contained a strong motion discontinuity between regions of rightward and upward local motion. In contrast, for the leftward moving object in the second example, the corner regions contained only a discontinuity between rightward motion and zero motion (there was no local upward component). This discontinuity is similar to the discontinuity near the centers of the left and right edges of the object. As a result, the selection activity was much more uniformly distributed across the entire edge of the object.

\section{Unit response properties}

The model described above can provide accurate velocity estimates for some visual stimuli, but does it match the way the brain solves this problem? A more critical test of the model is to compare the response properties of units in the local velocity and selection pathways to physiologically measured responses of cortical neurons. If the assumptions about the general approach used by the visual processing system are correct, and if the constraints on model reflect those in the visual system, then we should expect the responses of units in the model to match the response properties of neurons, at least qualitatively. We first review the physiological constraints embedded in the architecture of the model.

The first stage of our model was intended to correspond approximately to primary visual cortex. The properties motionenergy units in this first stage of the model are similar to the measured response properties of simple and complex cells in mammalian primary visual cortex (Tolhurst and Movshon, 1975; Holub and Morton-Gibson, 1981; Emerson et al., 1987, 1992; McLean and Palmer 1989, 1994; McLean et al., 1994; Qian and Andersen, 1994). The normalization of the motion-energy responses in the model reflects the saturating contrast response curves of cells in primary visual areas (Ohzawa et al., 1985; Heeger, 1992). The spatial and temporal response characteristics of the filters in the model, and the broader tuning in temporal frequency compared to spatial frequency, were based on mea- a)

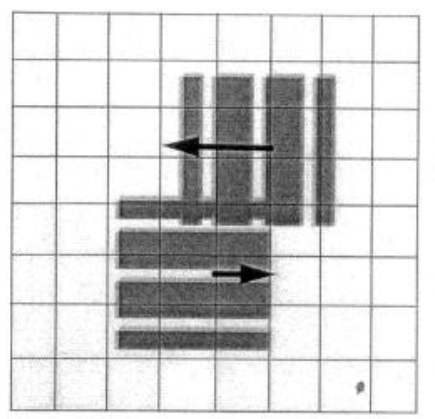

b)

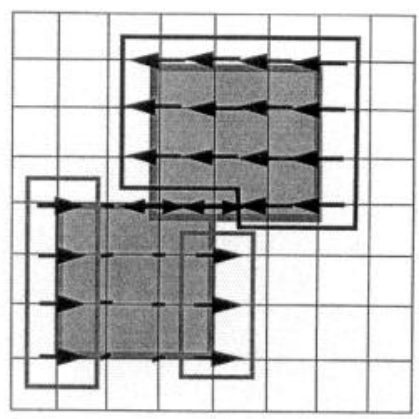

Figure 7. Illustration of local velocity and selection responses to partially occluding objects. $a$, The input to the model consists of two textured squares moving in opposite directions (one to the right at 0.25 pixels per frame, and the second to the left at 0.5 pixels per frame). The heavy arrows indicate the velocities of motion. $b$, Representation of the outputs of the local velocity and selection stages of the model for this stimulus. The arrows at grid points represent the output of the local velocity pool at that receptive field location. The light lines indicate the region of motion selected for the rightward moving square, while the dark line indicates a separate region of support for the motion of the leftward moving square. The local velocity estimatcs in the region of overlap show two directions of motion simultaneously and are rejected by the selection units.

sured responses of cells in primary visual cortex (Adelson and Bergen, 1985; Heeger, 1992). The inverse relationship between motion-energy filter center frequencies and receptive field sizes also matched relationships found in the retina and visual cortex (Hochstein and Shapley, 1976; Maffei and Fiorentini, 1977; Movshon et al., 1978; Andrews and Pollen, 1979).

The local velocity and selection stages of the model are primarily at the level of cortical area MT, although some of their functions may be distributed as well over area MST and parietal cortex. The response properties of units in these stages were not specified in advance, but several architectural decisions constrained their properties. The sizes of the receptive fields and the roughly retinotopic organization of the units were matched to the organization of early visual cortical areas (Maunsell and Newsome, 1987). The receptive fields in MT are on average 100 times larger than those found in V1 (Gattass and Gross 1981; Maunsell and Newsome, 1987) and the spatial scale of directional interactions were also larger (Mikami et al., 1986). In our model, the areas of the receptive fields for units in the selection and local velocity layers of the model were 81 times larger than receptive fields in the motion-energy layer. The feedforward "weights" of units into both the local velocity and selection pathway were constrained to be purely excitatory. Thus, the properties of the units in these networks were due primarily to excitatory inputs, with inhibitory effects being expressed only in the competitive renormalization used in all parts of the model (Eqs. 5, 8, and 9). These restrictions were based on both anatomical and physiological evidence from cortical microcircuitry (Douglas et al., 1989) and physiological experiments in primary visual cortex, suggesting that the primary role of inhibition may be nonspecific suppression and response saturation (Robson, 1988; Bonds, 1989).

The normalization used in our model was suggested previously to account for the gain control of neural responses in both V1 (Heeger, 1991) and MT (Snowden et al., 1991). The architecture proposed in our model makes two strong assumptions about the nature of this normalization in areas like MT. The 


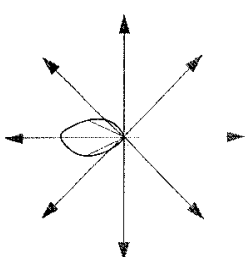

(a)

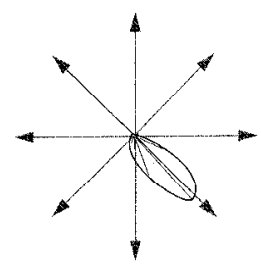

(d)

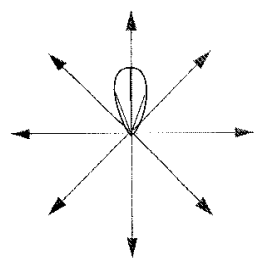

(g)

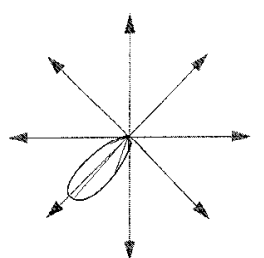

(b)

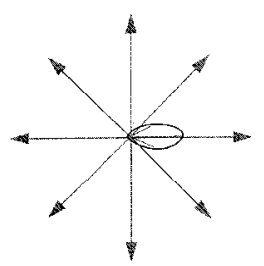

(e)

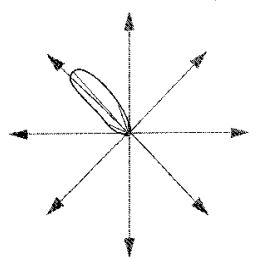

(h)

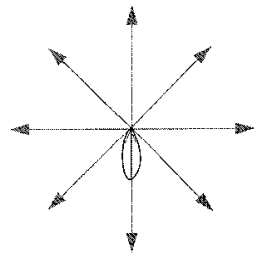

(c)

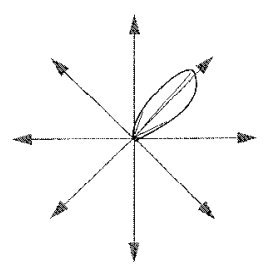

(f)

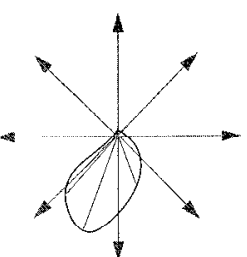

(a)

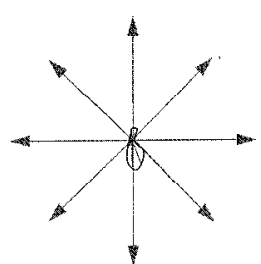

(d)

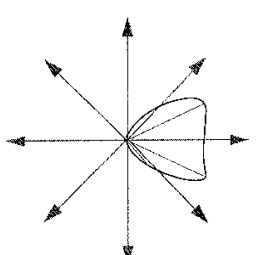

(b)

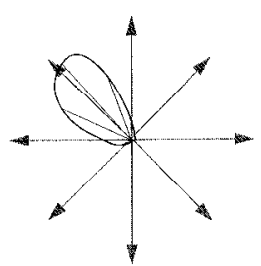

(e)

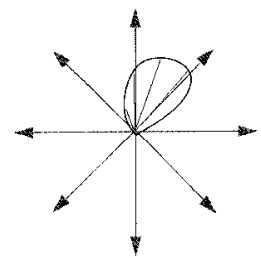

(c)

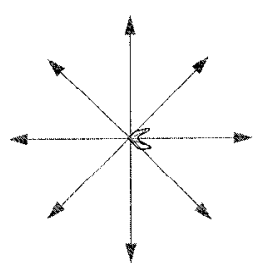

(f)
Figure 9. Directional tuning curves for selection units. $a-f$, The response of each six typical selection units is plotted in polar coordinates. Responses were determined using sinusoidal gratings. The unit depicted in $f$ exhibited a weak, bimodal response.

very close to zero for both local velocity units $(0.03)$ and selection units $(0.016)$.] The tuning curves for selection units showed considerably more variation. Selection units tended on average to have much broader tuning curves (average tuning bandwidth was $\left.84^{\circ}\right)$ and slightly lower index of directionality $(0.87)$. A small number of selection units showed a bimodal directional tuning curve (Fig. 9f).

The response of the model was also studied for flashed bars. The selection units tended to have maximal responses for bars oriented close to their preferred direction of motion, but local velocity units showed maximal responses for bars nearly orthogonal to their preferred direction of motion. Thus, if a velocity unit preferred an upwardly moving horizontal bar or horizontal grating, a flashed horizontal bar would also excite the unit, but a vertical bar would not. This is consistent with the local velocity unit integrating inputs from motion energy filters tuned for vertical upward motion. For a selection unit that preferred upward motion of an upwardly moving horizontal bar or horizontal grating, a flashed vertical bar would excite the unit more than a flashed horizontal bar. The flashed vertical bar produced a temporal frequency edge along the direction of preferred motion for the selection unit, which is consistent with the preference of the selection units for velocity discontinuities.

The broader directional tuning and similarity of orientation and direction tuning suggests that the selection units more closely resembled the "pattern" (Movshon et al., 1985) or Type II cells (Albright, 1984) found in monkeys and the local velocity units were more similar to the "component" or Type I cclls. The directional tuning bandwidths of both local velocity and selection units were sharper than the tunings found by Albright (1984), but in the same range as those reported by Maunsell and Van Essen (1983).

We explored the spatiotemporal frequency responses of selection and local velocity units using gratings oriented optimally for each unit, but spanning the range of temporal and spatial frequencies indicated above. Figure 10 shows the spatiotemporal frequency response of a velocity unit tuned to velocities near 0.5 pixels per frame. The response profile corresponded to a fairly sharp ridge passing through the origin with a particular orientation (Fig. 10a). This is characteristic for the responses of 
units that are tuned to a fairly narrow range of velocities over a broad range of spatial and temporal frequencies. Some cells with this type of broad spatiotemporal frequency response have been found in area MT (Newsome et al., 1983), but many cells appear to have narrower spatiotemporal frequency responses. The broad spatiotemporal frequency responses of velocity units in our model may represent the aggregate activity of pools of neurons with limited spatiotemporal frequency response. The insensitivity of velocity unit tuning to variations in temporal and spatial frequency began to break down only at the highest spatial and temporal frequencies (Fig. 10b). In general, the units in our model were tuned to velocity over a broader range of spatial and temporal frequencies than typically found in area MT, perhaps because there were many fewer units than MT cells to represent the same range. Thus, a single local velocity unit may represent, in area MT, a population of cells each of which is sensitive to velocity over a narrower range of spatial and temporal frequencies.

The spatiotemporal frequency responses of a selection unit is shown in Figure 11. This unit was paired in the model with the velocity unit plotted in Figure 10. The response of the selection unit was also a ridge in spatiotemporal frequency space, but, unlike the velocity unit, the selection unit ridge had a noticeable curve to it (Fig. 11a). The spatial frequency and temporal frequency sensitivities were in general more separable for selection units than for the local velocity units since they could be better approximated by products of purely spatial and purely temporal functions. In addition, the response of the selection unit showed a slight dip in the midrange frequencies, which was not observed in velocity unit responses. This curved response in frequency space caused a shift in the peak of the velocity tuning curve as the spatial frequency of the gratings was varied (Fig. $11 b$ ).

Nearly all of the selection units showed some degree of sensitivity of their velocity tuning to variation in spatial frequency, although Figure 11 represented an extreme case. The consistency of this sensitivity to spatial frequency suggests that it provided some computational advantage for the selection units, but the exact nature of this advantage is not yet clear. Many MT neurons also show some sensitivity of their velocity tuning to spatial frequency (Maunsell and Newsome, 1987), but this variation has not been systematically correlated with other types of variation (such as tuning width).

Another important qualitative difference in the responses of local velocity and selection units was apparent when gratings were either restricted to the receptive field region or presented across the entire visual field. The responses of local velocity units were not affected, but the responses of selection units were strongly suppressed when the grating was presented to the entire visual field rather than just to the receptive field of a unit. (The receptive field size was determined by the fan-in of the feedforward connections to the unit.) The suppression of response due to motion in regions surrounding the selection unit receptive

Figure 10. Spatiotemporal response properties of a representative local velocity unit. The unit was tuned to a velocity of 0.5 cycles per frame. $a$, Contour plot of responses to sinusoidal gratings as a function of spatial and temporal frequencies. Contour lines are spaced at response intervals of 0.2 and the maximal response at the middle pair of lines is $0.8 . b-$ $d$, Velocity tuning for gratings with three different spatial frequencies. The velocity tuning is approximately independent of spatial frequency, but the peak is shifted slightly to a lower velocity at the high spatial frequencies $(b)$. a)

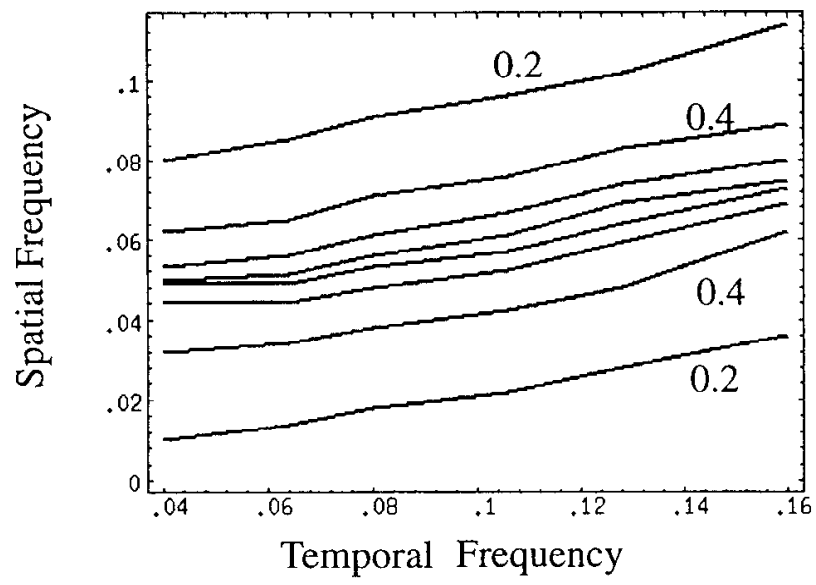

b)

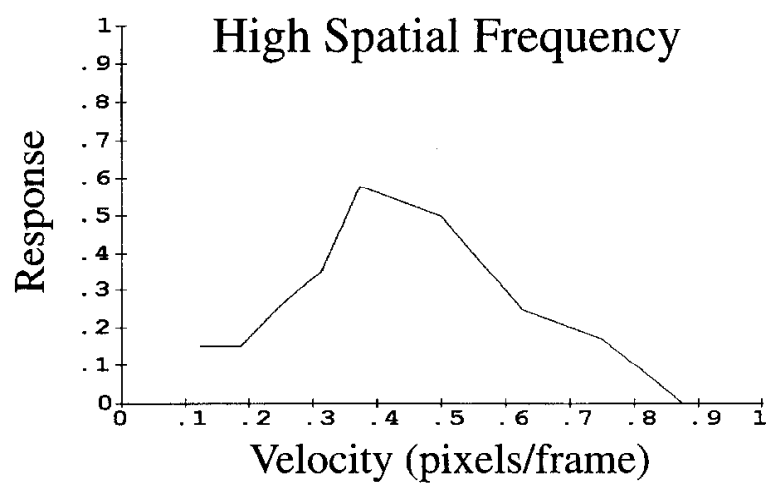

c)

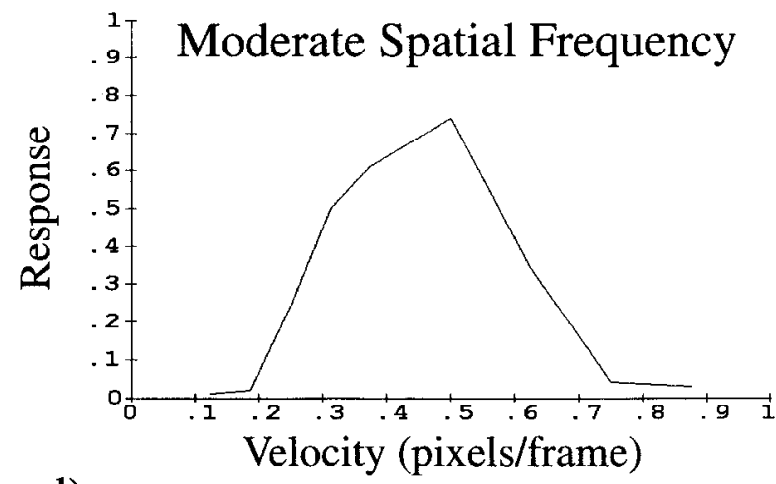

d)

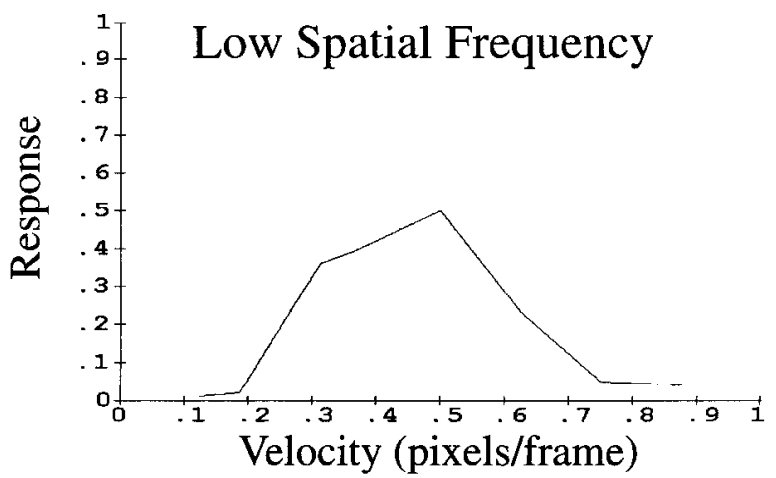


a)

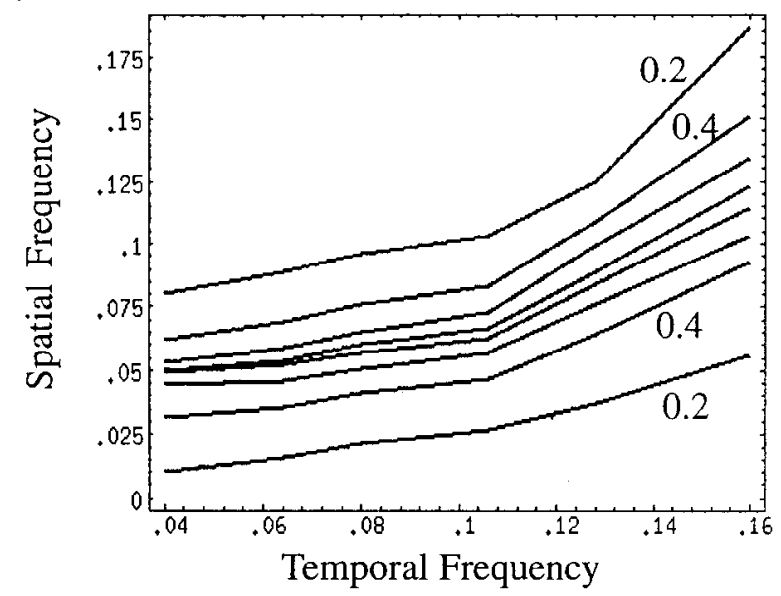

b)

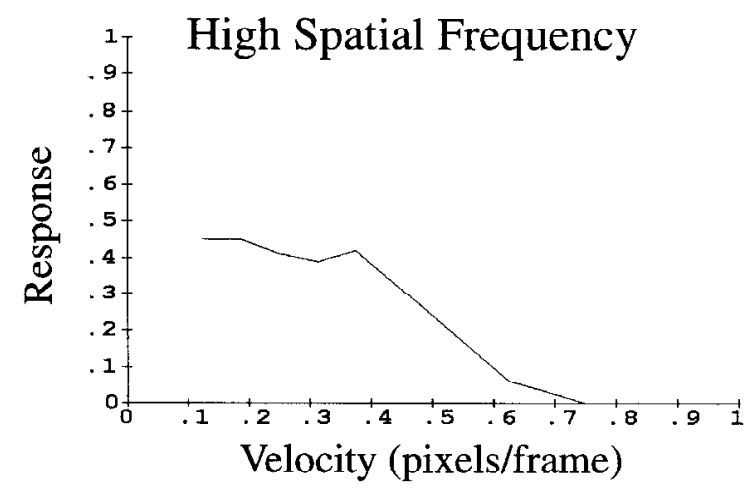

c)

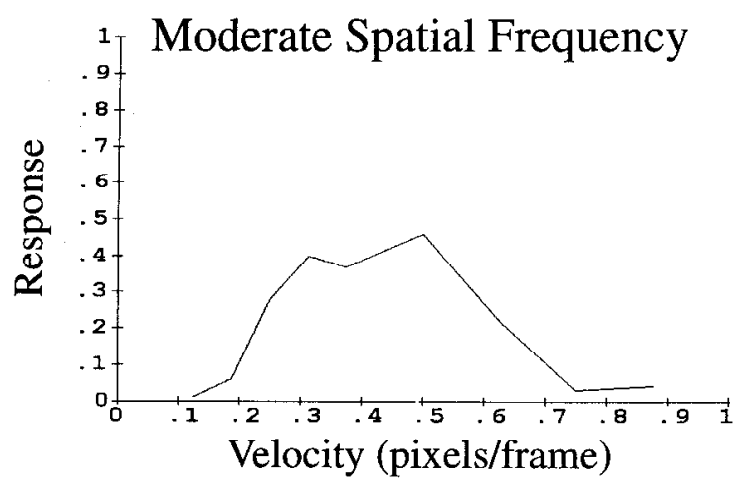

d)

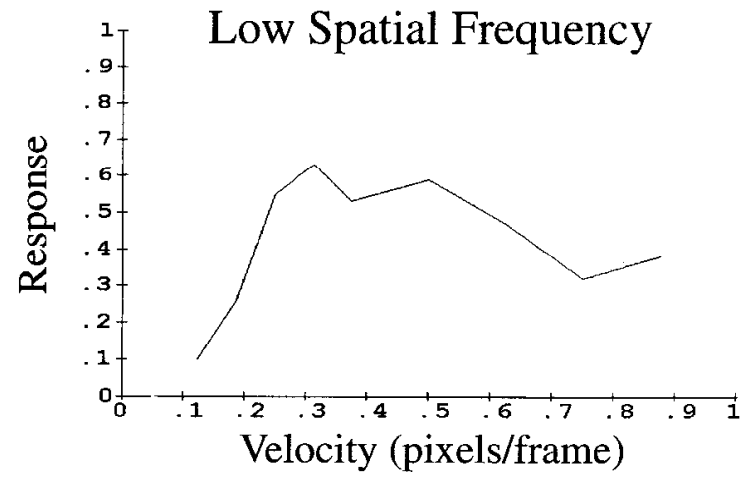

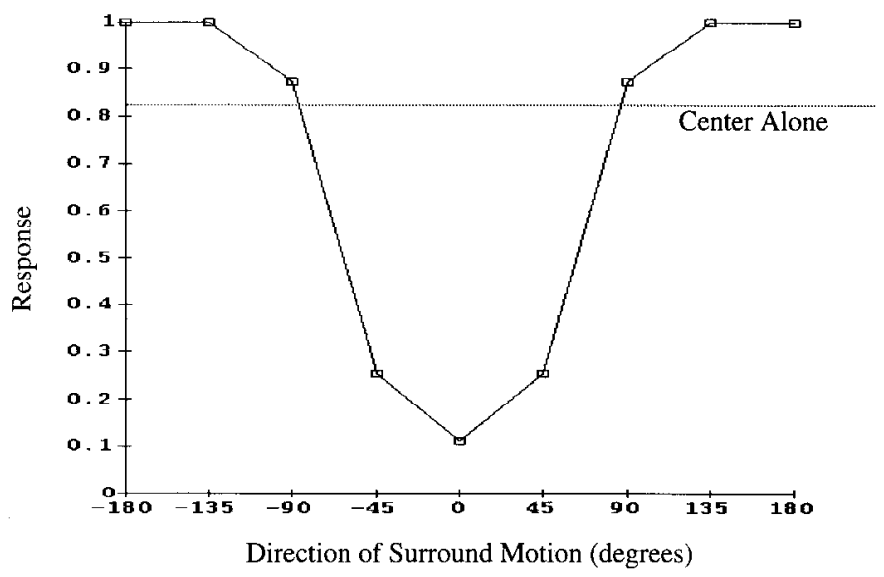

Figure 12. Effect of a sinusoidal grating presented outside the receptive field of a selection unit responding to an optimal sinusoidal grating within its receptive field. The dashed line shows the response of the selection unit to the grating within the receptive field alone. The orientation of the surround grating is measured relative to the orientation of the grating within the receptive field. When the direction of surround motion matched the motion within the receptive field, there was strong inhibition of the response. The inhibition decreased as the directions of motion became more mismatched and became facilitatory when the difference in orientation was greater than $90^{\circ}$.

field was strongest when the surrounding motion matched the optimal motion for eliciting a response in the selection unit's receptive field and decreased as the surround motion differed more from the optimal motion for the unit (Fig. 12). In fact, motion in a direction more than $90^{\circ}$ away from the optimal direction for the selection unit tended to facilitate the response of the selection unit. Modulation of the receptive field response by the nonclassical surround in area MT with these characteristics was first reported by Allman et al., 1985 (see Discussion).

The suppressive surround response of selection units can be explained by the competitive interaction of similarly tuned selection units across the entire visual field. The model has effectively a fixed amount of selection activity for a particular velocity that must be distributed across the visual field. When a velocity is present in only one region of the visual field, the selection activity for that velocity can be concentrated at that location. When the velocity is present across the entire visual field, the selection activity must be shared across all locations, and any one location receives only a small portion of that activity.

The substructure of the receptive fields in area MT were studied by Snowden et al. (1991) whose stimuli consisted of random dots moving within the classical receptive field. One field of dots always moved in the preferred direction of the cell, while a second field, if present, moved in a different direction, pro-

Figure 11. Spatiotemporal response properties of a representative selection unit. $a$, Contour plot of responses to sinusoidal gratings as a function of spatial and temporal frequencies. The contour lines are labeled as in Figure 10. The ridge of the response function is curved, bending upwards on the right side of the plot, unlike the responses of velocity units in Fig. $10 . b-d$, Velocity tuning for gratings with three different spatial frequencies. There is a double peak at moderate spatial frequencies and the tuning was much broader for higher and lower spatial frequencies. This selection unit corresponds to the velocity unit shown in Figure 10. 


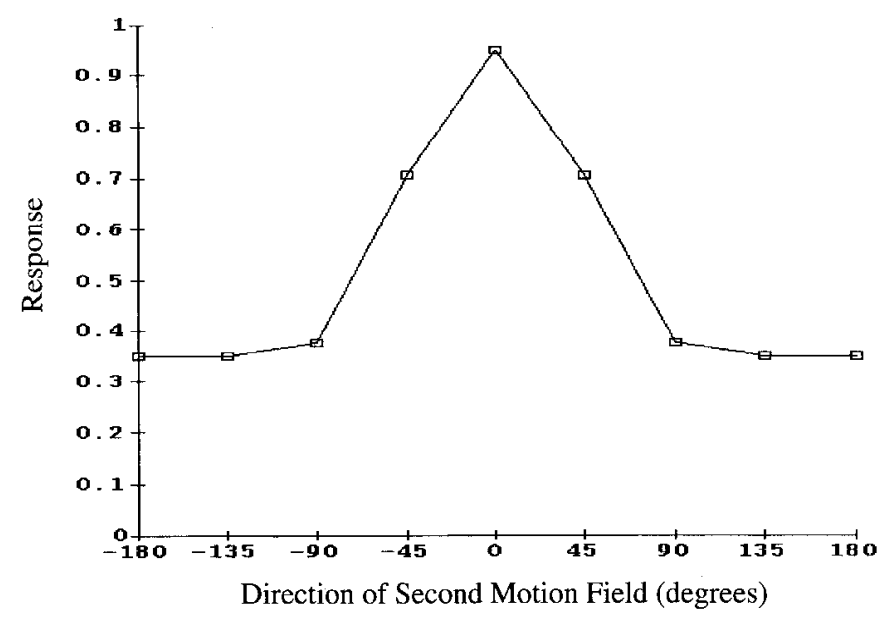

Figure 13. Response of a local velocity unit to a transparent random dot stimulus consisting of two fields of random dots moving within the receptive field. The first field was chosen so its motion produced a maximal response from the unit. The response is shown as a function of the direction of the second field relative to the first. The presence of a second motion always suppressed the response of the unit, with this suppression saturating for motions orthogonal to the preferred direction of motion (at which point the local velocity pool had a bimodal activity distribution).

ducing two distinct motions simultaneously within the cell's receptive field. When similar stimuli were presented to the local velocity units, their responses were qualitatively similar those observed by Snowden et al. (Fig. 13). The presence of a second motion in the receptive field always suppressed the response of a local velocity unit, with the degree of suppression increasing until the second motion was orthogonal to the preferred direction of the unit, after which it was constant. These response characteristics can be explained by the local competition within each pool of velocity units, since the model was based on a distributed representation of velocity with units representing overlapping ranges of velocities. The suppressive effect of motions close to the preferred motion for the unit were less than that for motions that were more different.

Snowden et al. (1991) suggested that the suppressive effects seen in the MT cells were best modeled by a divisive inhibition with saturation. The soft-maximum operation used in the model produced precisely this type of suppression (increasing the number of dots in the nonpreferred direction caused the slope of the response as a function of dot density in the preferred direction to decrease, but the response always saturated at the same level). The model made the additional prediction that a second random-dot pattern moving in a cell's preferred direction at a speed significantly different from the optimal speed for the cell would also have a suppressive effect on the cell's response to an optimal stimuli.

\section{Psychophysical comparisons}

Motion processing in the human visual system has been studied extensively with psychophysical experiments (Levinson and Sekuler, 1976; McKee 1981; McKee and Nakayama, 1984; McKee et al., 1986; Braddick, 1993); recently, physiological correlates of several psychophysical motion phenomena have been found in recordings from cells in area MT of awake primates (Newsome et al., 1989; Albright, 1992; Britten et al., 1992; Stoner and Albright, 1992; Dobkins and Albright, 1993).
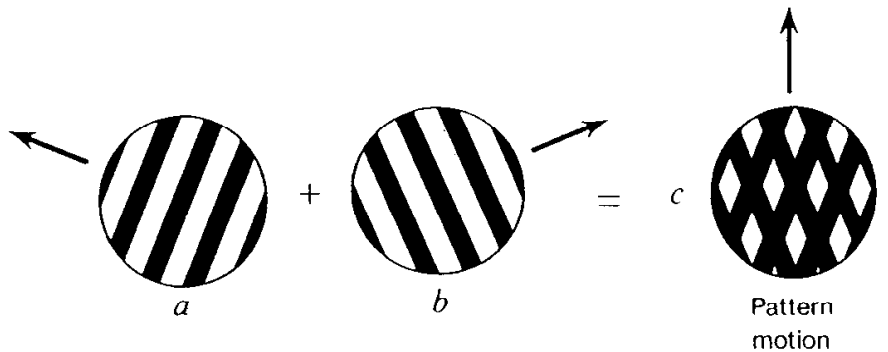

Figure 14. Plaid pattern stimuli composed of two superimposed drifting gratings. $a$ and $b$, When either grating was presented alone, its direction of motion is reported as shown by the arrows. $c$, When the two gratings were superimposed, they cohered and the pattern appeared to move up (arrow), which was in a direction different from that of either component grating (From Stoner et al., 1990.)

Neurons with similar properties have been found in area MST and other cortical areas, but we focus here on two recent series of experiments performed in area MT that combine single-unit recording and psychophysical studies.

Plaids. Moving plaid patterns have been used to study how the visual system integrates multiple motion signals into a coherent motion percept (Adelson and Movshon, 1982; Ramachandran and Cavanagh, 1987; Welch, 1989; Stoner et al., 1990; Stoner and Albright, 1992b; Wilson et al., 1992). These stimuli consist of two independently moving gratings that are superimposed (Fig. 14). When human observers are presented with either grating alone (Fig. $14 a . b$ ), they always reliably report the motion of the grating. However, when two identical gratings are superimposed (Fig. 14c), rather than see the two independent motions, most observers see the two gratings cohere and form a single pattern moving in a direction different from either of the gratings. The direction of pattern motion usually corresponds to the velocity of the intersection of the two component gratings, usually referred to as the intersection of constraints (IOC) direction, as shown in Figure 1 (Adelson and Movshon, 1982), although this may not always occur (Wilson et al., 1992; Rubin and Hochstein, 1993). Cells that respond in the direction of pattern rather than component motion were first found in area MT and are more common in MT than V1, which suggests that MT may be site of integration for pattern motion (Movshon et al., 1985; Rodman and Albright, 1989; Ferrera and Maunsell, 1991).

Stoner et al. (1990) have demonstrated that the tendency to see coherent pattern motion can be affected by altering the luminance of the intersection between the two gratings. Some cells in area MT also tend to respond to either the direction of pattern or component motion, depending on the luminance of this intersection region (Stoner and Albright, 1992). The visual stimuli used by Stoner and Albright consisted of two square wave gratings with thin bars on a lighter background (Fig. 15a). Squarewave gratings were used, since this allowed the luminance of the intersection regions to be varied while leaving the luminance of the bars and the background the same. Figure $15 b$ shows an expanded view of the intersection region, in which the intersection has been labeled $A$, the bars in the two gratings were labeled $B$ and $C$, and the background was labeled $D$. In the psychophysical and physiological experiments, regions $B, C$, and $D$ were held at a constant luminance (with the luminance of $B$ and $C$ identical).

Stoner et al. manipulated the luminance of region $A, L_{A}$, and 

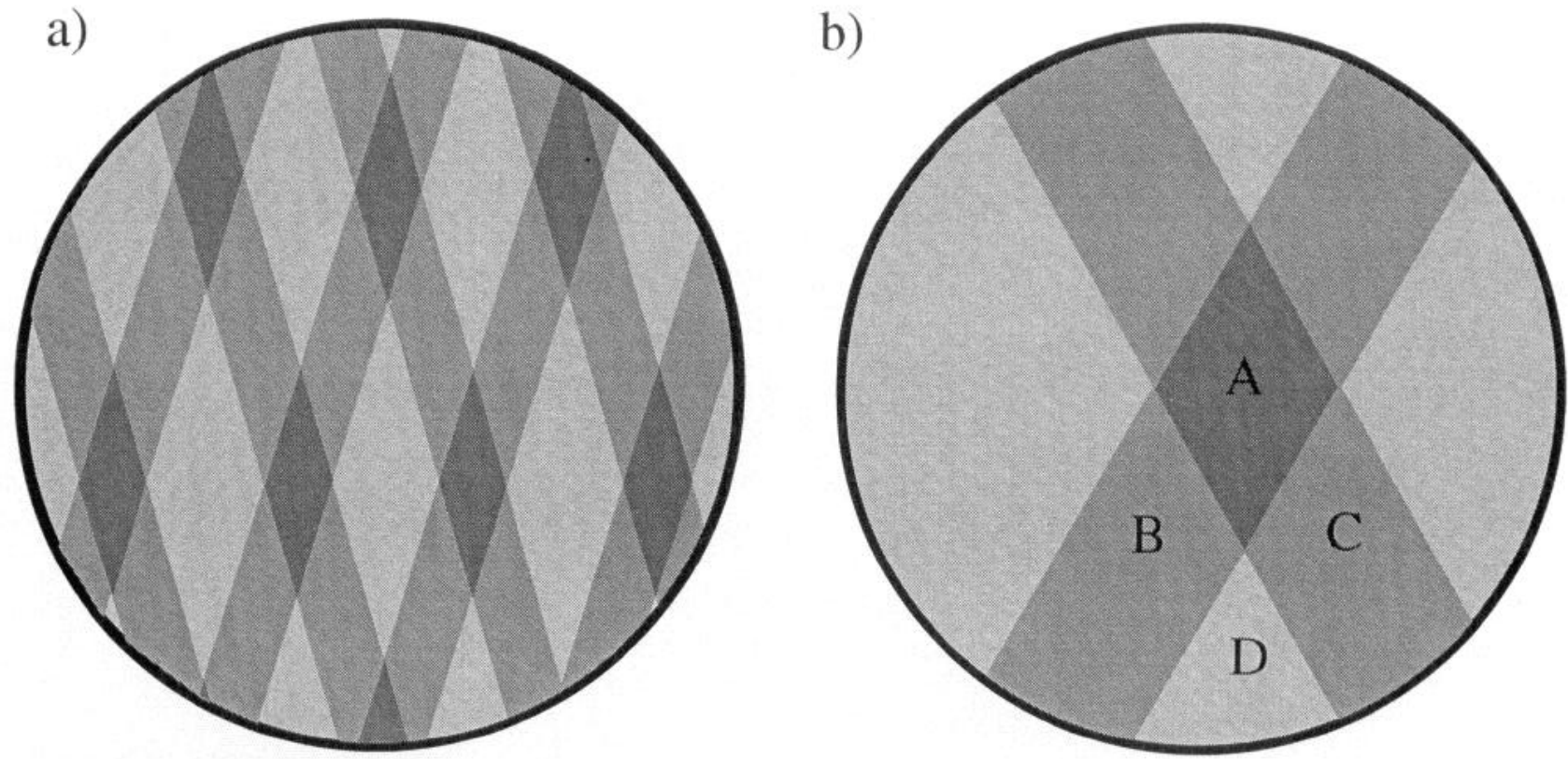

Figure 15. Plaid pattern stimuli used by Stoner et al. (1990) to demonstrate transparent motion. $a$, The plaid patterns were composed of two square wave gratings of gray bars on a lighter background. $b$, Detail of the intersection of the pattern. The intensity of the region labeled $A$ was varied, while the intensity of regions $B-D$ were held constant. Depending on the intensity of $A$, the observer would see either two drifting gratings slipping through each other or a single coherent pattern motion moving up.

showed that there is a range of luminances for which humans see one transparent grating lying on top of the other and reliably report the presence of the two grating motions rather than the coherent pattern motion (Fig. 16a). This "transparency zone" was bounded at one end by the condition in which the luminance of regions $A$ and $C$ were identical, which corresponded to one grating completely occluding the background and the other grating (left vertical line in Fig. $16 a, b$ ). The bound at the other end was defined by

$$
\frac{L_{A}}{L_{B}}=\frac{L_{C}}{L_{D}},
$$

which implies that one of the gratings was physically transparent, having no reflectance, and merely attenuated the light from regions $C$ and $D$ (right vertical line in Fig. 16a,b). This type of multiplicative transparency is also characteristic of shadows.

We repeated the experiment of Stoner et al. by presenting the model with a series of plaid patterns consisting of two square wave gratings in which the luminance of the intersection region of the plaid pattern was varied systematically through a series of values that spanned the transparency zone. The luminance of the intersection region for the model was computed by taking the intensity of the intersection region and dividing it by the maximum input intensity (255). A value of 1.0 on this scale corresponds to a pure white, a value of 0.0 corresponds to a pure black, and values in between correspond to various shades of gray. The response of the output units in the model varied nonlinearly with the luminance of the intersection region. In order to compare this variation to the psychophysical results, we used a measure of the percentage of response corresponding to the component motion (Stoner et al., 1990):

$$
\mathrm{PC}=50\left(1-\frac{a_{P}}{I_{P}}+\frac{a_{C}}{I_{C}}\right),
$$

where PC stands for percentage component motion, and $I_{P}$ is the ideal response to pattern motion, $a_{P}$ is the actual amount of pattern response, $I_{C}$ is the ideal response to component motion, and $a_{C}$ is the actual response to component motion. $\left(a_{P}, I_{P}, a_{C}\right.$, and $I_{C}$ are strictly positive and $<1 ; a_{C} \leq I_{C}$ and $a_{P} \leq I_{P}$. Thus, PC lies between 0 and 100.) The ideal component response was computed by presenting the gratings to the model independently, and adding together all of the responses of the output units above a threshold of $1 / 33$ to get a single scalar value. This threshold was the approximate resting state of the output units. The actual component response was computed by adding together the activity in all of the output units that were above threshold in the ideal case (typically around four units). The ideal pattern was a pattern of intersecting bars (with intersection regions having the same luminance as the bars) that looked like a plaid pattern when viewed statically. This entire pattern was moved in the IOC direction of the original plaid stimuli. The ideal pattern response was computed by adding together the activity of all the output units above threshold. The actual pattern response was computed by adding together the activity in the output units that had been above threshold in the ideal pattern response (typically around two units).

$\mathrm{PC}$ is not the most direct measure of the response of the model, but it captures two important effects that are present in the comparable psychophysical experiments. First, the PC measure takes into account the effects of random variation; units in the model are noise free so the response to any particular input pattern is deterministic. A simple winner-take-all strategy based on the strength of the output of units tuned to component motion versus the output of units tuned to pattern motion would always pick the larger response even when the two responses were nearly identical in value. In the presence of random variation, however, when the two responses are nearly equal in value, we would expect each response to win roughly half of the 
a)

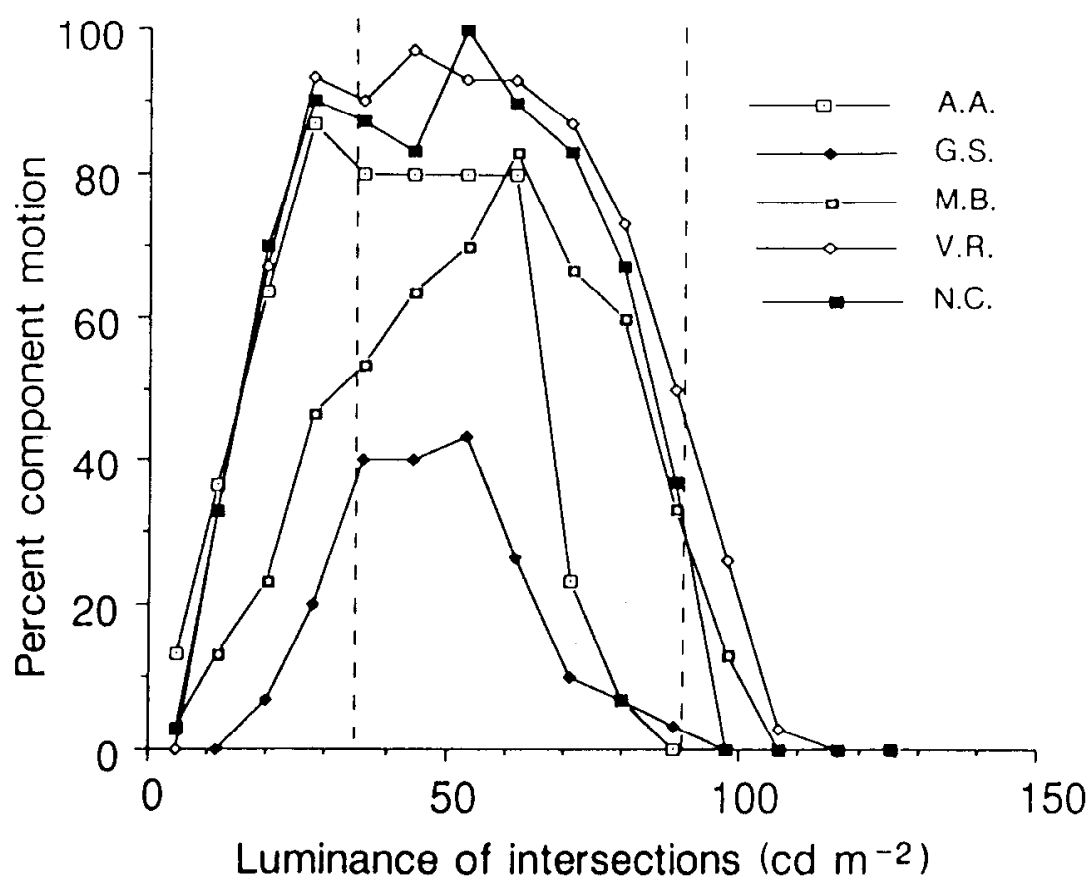

b)

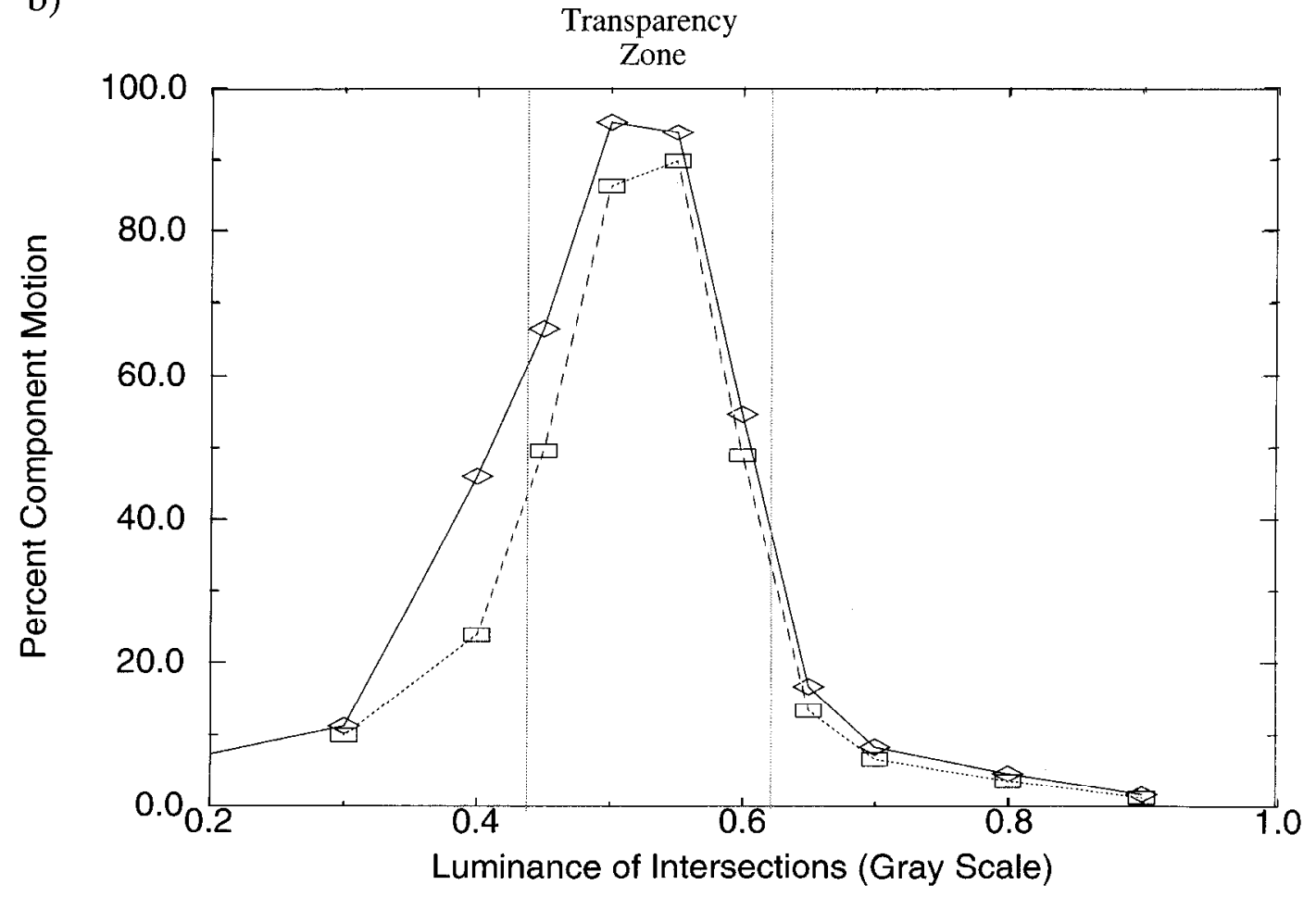

Figure 16. Responses of human subjects and model to plaid patterns as a function of intensity of the intersection region. $a$, Psychophysical results for five human subjects from Stoner et al. (1990). Within the transparency zone defined by the dashed lines, subjects generally perceived two independently moving component gratings, but, outside this region, subjects tended to perceive coherent pattern motion. $b$, Responses of selection model to similar plaid patterns. The solid line represents responses to plaid patterns made from gratings inclined at $135^{\circ}$ like those of Stoner et al. The dashed line represents responses to plaid patterns made from gratings inclined at only $90^{\circ}$. For both types of plaid, there was a transparency zone (dotted vertical lines) within which the responses of the model were more often consistent with two separate component motions. Outside this transparency zone, the model tended to report only a single pattern motion. The $\mathrm{x}$ axis in $a$ rcprcsents the physical luminance of patterns seen by human observers, while the $\mathrm{x}$ axis in $b$ represents the intensity of an input pixel divided by the maximum possible intensity of an input pixel. On both scales, the brightness or whiteness increases as one moves to the right. The actual mapping between the scales is arbitrary, but on both graphs the left vertical line corresponds to the luminance of regions $A$ equaling the luminance of region $C$ in Figure 15, while the right vertical line corresponds to $L_{A} / L_{B}=L_{C} / L_{D}$. 

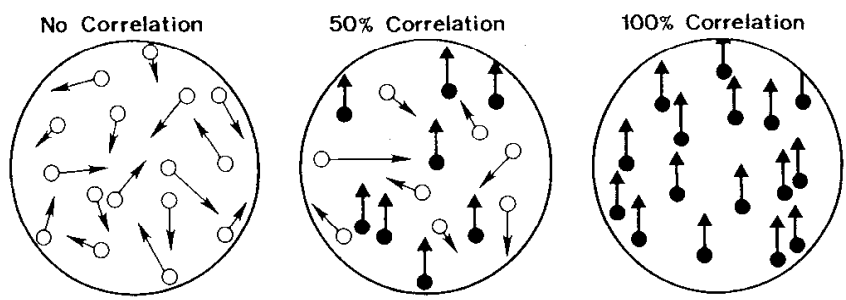

Figure 17. Dynamic random-dot stimuli used by Newsome et al. (1989). Left, No correlation. The locations of all dots in the next frame were chosen randomly. There is no net motion in this type of display, although there are many instantaneous motion events from chance juxtapositions of dots. Middle, Fifty percent correlation. Half of the dots were replaced in random locations while the other half were replaced a fixed displacement from their current locations. One perceives net upward motion against a background of dynamic noise. Right,) One hundred percent correlation. The locations of all dots in the next frame are a fixed displacement from there location in the current frame. One observes net upward motion of the entire collection of dots. (From Newsome and Pare, 1988.)

time. Similarly, $\mathrm{PC}$ is near $50 \%$ when the pattern and component responses are nearly equal. The second reason for using PC is that the psychophysical experiments are choice experiments: the subject must decide whether the percept falls in one of two categories. The PC measure also compares the actual responses to ideal responses in order to decide the category for the actual response. This is especially important for plaid stimuli since we did not train the model with similar stimuli and, therefore, had no a priori expectation of what the model response corresponding to these stimuli should be. For example, the activity of units in the ideal pattern response were generally weaker than the component response, so simply using unit response values would lead to misleading results.

$\mathrm{PC}$ is plotted as a function of the luminance of the intersection region in Figure $16 b$ for two different sets of stimuli. The solid line corresponds to plaid stimuli identical to those used by Stoner et al., with a $135^{\circ}$ angle between the two components of the plaid and a duty cycle of $28.6 \%$, as defined by Stoner et al. (1990). The dotted line corresponds to plaid stimuli which have only a $90^{\circ}$ angle between the gratings and a duty cycle of $33 \%$. There is a good match between the psychophysical results shown in Figure $16 a$ and the responses of the model to both sets of plaid stimuli. In both cases, the highest probability for seeing separate component motions occurs within the transparency zone, with the curve dropping off quite steeply outside this zone. The region of transparent responses is somewhat narrower for the $90^{\circ}$ plaids, and this is also consistent with psychophysical results. When the angle between the plaid patterns in the psychophysical experiments decreased, subjects more often fused the two motions into a single motion and the region over which transparent motion was reported became narrower (Stoner 1992).

There was a significant difference in the distribution of support across the visual field when the model reported pattern versus component motion. For gratings in which the percentage component motion was less than $20 \%$, more than $70 \%$ of the total support for motion was concentrated on the regions surrounding the intersections of the two gratings. (The total support for motion in the scene included the support for all global velocity units that are active at a level above 0.05 .) Conversely, for gratings in which the percentage component motion was more than $80 \%$, more than $65 \%$ of the total support for motion in the scene was concentrated on the portions of the gratings which were outside the intersection regions. Thus, perception of com- ponent or coherent motion by the model seemed to depend primarily on whether support for global motion processes was concentrated on the intersection regions or away from them.

The amlount of support assigned to the intersection regions of the plaid patterns depended on the contrast differences between the intersections, bars, and background. Two opposing factors determined the amount of selection unit activation in the intersection regions. The intersection regions contained motion signals at two different orientations, which tends to increase selection unit activity (Fig. 5, pattern 2). On the other hand, the model had been trained to ignore motion signals created by the interactions between two different objects moving in different directions (Fig. 5, pattern 4; Fig. 7).

Many of the training examples included physical transparency, and, based on this exposure, the selection units discounted motion energy discontinuities created by transparent interactions. These types of discontinuities could be identified by the contrast ratio across the discontinuity. When the contrast between the intersection region and bars of the plaid pattern fell in this intermediate range, selection unit activity in the intersection regions was reduced so support for motion was now stronger along the contrast edges between the bars of the components and the background. Exposure of the model to examples of transparent interactions during training was critical to the ability of the model to report component rather than pattern motion: models that were trained on a reduced training set that lacked transparency always reported pattern motion for plaid patterns. However, the model could generalize to novel transparent stimuli. The training set included no examples of plaid patterns, and all the examples of transparent interactions were between objects moving in opposite directions.

In humans, the coherence of plaid patterns can also be affected by spatial frequencies of the two components (Ramachandran and Cavanagh, 1987; Welch 1989). If the component frequencies are very different, human observers always see two separate motions rather than a single cohcrent motion. We performed similar experiments with the model and found that the model fails to see coherent motion when the difference in the ratio of the component spatial frequencies is an octave or greater (see Nowlan and Sejnowski, 1994, for details). This is similar to human performance on the same stimuli. The failure of the model to report coherent motion in plaids with very different spatial frequencies can be traced to a failure to integrate across very different spatial frequencies in the local velocity stage of the model. The range of spatial frequencies for which plaid patterns cohere corresponds very well to the range of frequencies over which an individual local velocity unit responds.

Random dots. We also used dynamic random-dot displays to probe the model (Braddick, 1974; Morgan and Ward, 1980; Nakayama and Tyler, 1981; Williams and Sekuler, 1984; Newsome and Pare, 1988; Britten et al., 1992). Random-dot displays remove most high level cues (such as particular geometric features) which could be tracked between frames to infer motion. It is also easy to ensure that parameters such as spatial and temporal frequency content, mean luminance, and contrast are evenly balanced in the visual display. Dynamic random-dot stimuli can have more than one flow field represented within the same region, which challenge traditional vision models that attempt to integrate information from a velocity flow field using smoothness constraints. In a dynamic random-dot display, there are no regions of consistent motion and the velocity of neighboring dots is in general very different. 
The stimuli used in our experiments were based on the dynamic random-dot stimuli used by Newsome and Pare (1988). They prepared a range of stimuli in which the strength of a coherent motion signal varied relative to random dynamic background noise. The stimuli consisted of a field of randomly distributed dots with a very limited life span. When a dot vanished, it was immediately replaced with a new dot, so the average dot density always remained the same. At one extreme, referred to as the no correlation condition, the location of the replacement dot was chosen entirely at random. Perceptually, the overall impression of this display was of a field of twinkling visual noise. Many momentary motion events are generated by fortuitous pairings of random dots, but no there is no consistent motion in any direction (Fig. 17, left). In the other extreme condition, each replacement dot was positioned at a fixed displacement and direction from the dot it was replacing. All the random dots were then perceived as moving at the same speed and in the same direction (Fig. 17, right).

Intermediate stimuli were prepared in which a proportion of the dots were moved coherently while the remaining dots were replaced with new dots in random locations (Fig. 17, middle). The group of dots moving coherently were perceived as moving against a background of random noise. By varying the percentage of dots that moved coherently, the strength of the motion signal relative to the noise was smoothly varied. For these intermediate stimuli, although a fixed proportion of the dots were moved coherently at each replacement, the dots that moved coherently were chosen randomly each time they were replaced. It was highly unlikely that the same dot moved coherently for any extended period. Thus, the location of coherent motion signals was constantly varying, and generally each such coherent signal was surrounded by many other signals corresponding to different motions (Fig. 17, middle).

Newsome and Pare (1988) showed that in a forced choice task, rhesus monkeys were able to reliably identify the direction of motion in these random-dot stimuli even when fewer than $5 \%$ of the field of dots moved coherently. In subsequent studies, Newsome et al. (1989) and Britten et al. (1992) computed psychometric functions for the ability of a monkey to correctly identify the direction of motion as a function of the percentage of dots moving coherently and showed that these psychometric functions were closely matched by "neurometric" functions computed from the responses of single MT neurons (Fig. 18a). Some MT neurons had information in their firing rate that was as reliable as the behavior of the monkey.

We examined the response of our model to dynamic randomdot displays in which the percentage of correlated dots varied from $0 \%$ to $100 \%$. Our stimuli were constructed in the same way as those of Britten et al., and consisted of 64 discrete frames with dots replaced at each frame. Between adjacent frames, a portion of the dots were displaced coherently and the remainder were replaced at random locations.

Since the psychometric functions in the study of Britten et al., 1992, were hased on a two-way forced choice paradigm, we presented stimuli in which the patch of coherent dots (if it existed) moved either right or left. Five leftward and five rightward stimuli were presented at coherence levels of $0.5,1,2,5$, $10,20,50$, and $100 \%$. The following performance measure was defined for each rightward stimuli:

$$
\mathrm{PC}_{R}=100 \frac{a_{R}}{a_{R}+a_{L}},
$$

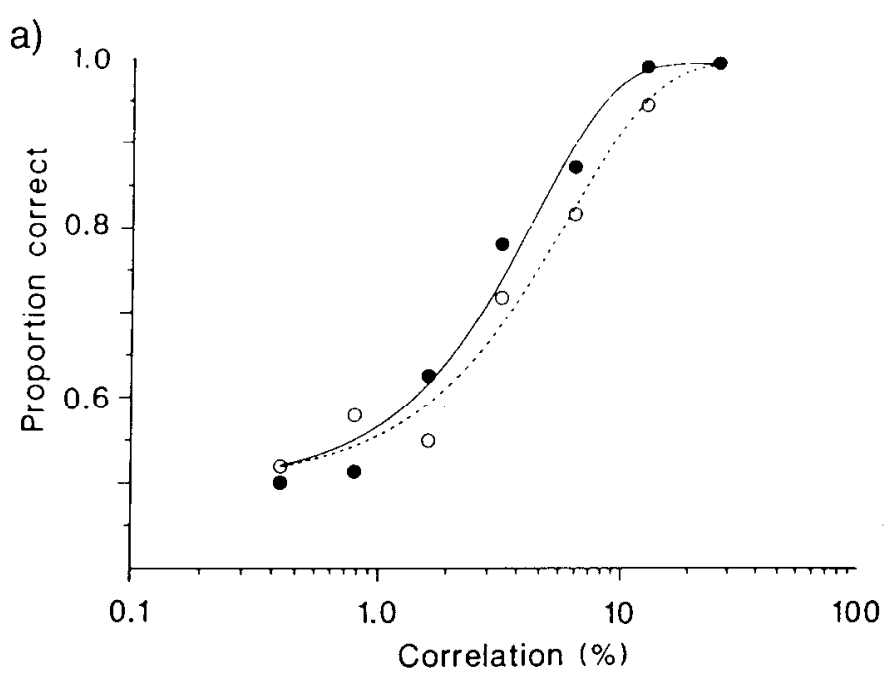

b)

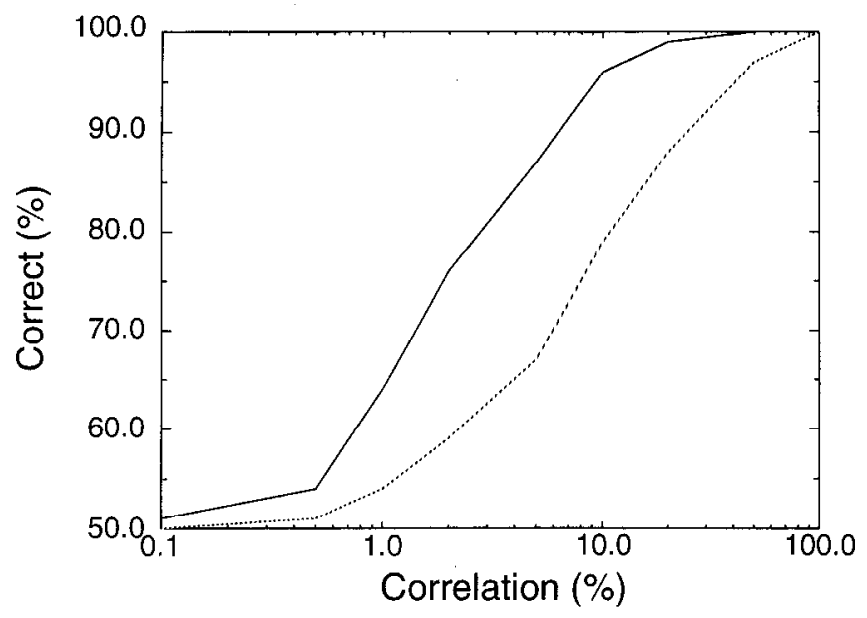

Figure 18. Responses of rhesus monkey and model to dynamic random-dot stimuli. $a$, Percentage of correct responses from a monkey in a forced choice paradigm as a function of percentage coherence in the stimuli ( $\mathrm{O}$ and dashed line). Neurometric decision function computed for an MT neuron from this monkey (- and solid line). (From Newsome et al., 1989.) $b$, Percentage of correct responses from model in a forced choice paradigm as a function of percentage coherence in similar stimuli. Solid line is the performance of intact model and the dashed line is the performance of model when selection pathway was disabled (see text). The primary effect of disabling the selection pathway was to shift the detection curve to the right, raising the threshold for detection from $4 \%$ to $18 \%$.

where $\mathrm{PC}_{R}$ indicates the percentage of correct responses for a rightward moving stimulus, $a_{R}$ is the total global rightward activity (computed by summing the activity of all four global output units representing rightward motion), and $a_{L}$ is the total leftward activity. This measure is similar to the PC measure defined for the plaid patterns and is, again, intended to model forced choice in the presence of random variation. If $a_{R}$ and $a_{L}$ are nearly equal, $\mathrm{PC}_{R}$ is close to $50 \%$, indicating that a winnertake-all decision in the presence of noise would select rightward motion roughly half of the time. A symmetric measure is defined for the trials involving leftward motion. These measures were averaged across the five leftward and five rightward trials for each coherence level to compute a percentage correct response at a given coherence level. 
The percentage of correct responses is plotted as a function of coherence level in Figure 18b, and the psychometric response curve from Britten et al. (1992) is shown in Figure $18 a$ for comparison. The general shape of the response functions and the detection thresholds are very similar, with the model performing slightly better than the monkey. (The monkey identified the direction correctly on $82 \%$ of trials at a coherence level of $6 \%$. The model attained $85 \%$ correct identification at $4 \%$ coherence.) The difference between the model and the monkey is not statistically significant.

During presentation of these dynamic random-dot stimuli, the region of support for the coherent motion in the selection network was highly discontinuous, and could change dramatically between successive groups of frames. These changes in the support region reflected the changes in locations of coherently moving dots in the input stimuli. These discontinuous and dynamic support regions were quite unlike what one would expect from a regularization scheme that employed smoothness constraints, and raised the question of whether the selection pathway has a critical role in processing these stimuli. Further analysis of the selection network in Nowlan and Sejnowski (1994) revealed that chance clustering of coherent dots enabled the selection network to segment regions that had higher than average signal-to-noise. If this explanation is also correct in primates, then performance should degrade if displays are designed to prevent clustering of coherent dots.

To further address this question, we ran the same stimuli through the model while disabling the inputs to the selection pathway. With the selection stimuli disabled, all regions of an image were weighted equally, and global velocity signals are formed from simple averages over all local velocity signals. Figure $18 b$ displays the percentage of correct responses as a function of percentage coherent dot motion when the selection pathway was disabled. 'The primary effect of disabling the selection pathway was to shift the response curve further to the right, raising the detection threshold for the coherent motion to $18 \%$ coherence to identify the direction correctly 85 ) of the time. This represented an over fourfold increase in detection thresholds.

The increase in the model's detection threshold was the same order of magnitude of increase observed in the detection thresholds of monkeys with lesions to area MT (Newsome and Pare, 1988). Rhesus monkeys with intact area MT reliably identified the direction of motion in a dynamic random-dot stimuli when fewer than $5 \%$ of the field of dots moved coherently. However, when area MT was lesioned detection thresholds increased to between $20 \%$ and $40 \%$ (four to eight times greater). More experiments need to be done comparing the performance of the lesioned monkey with that of the lesioned network to establish their similarity.

\section{Discussion}

Motion perception requires the visual system to satisfy two conflicting demands: first, signals from neighboring regions of the visual field need to be spatially integrated to overcome noise, and second, sensitivity to small velocity differences across space should be preserved to segment regions corresponding to different objects (Braddick, 1993). These demands were satisfied in our model by two separate networks, one that computed the local velocity estimate and a second that selected regions where reliable velocity estimates are possible. The outputs of these two networks were combined to produce reliable estimates of the velocities of objects without assuming spatial continuity.

\section{Comparisons with previous models}

Previous models of motion processing that assumed spatial continuity of the flow field were limited in their ability to handle occlusion and transparency (Marr and Ullman, 1981; Adelson and Movshon, 1982; Hildreth, 1984; Heeger, 1987; Grzywacz and Yuille, 1990; Sereno, 1993). A few models have attempted to find motion boundaries. For example, the velocity gradient has been used by Koch et al. (1989) to determine the boundaries between regions with different uniform velocities and by Smith and Grzywacz (1993) to determine where to apply a winncrtake-all operation. Jasinchi et al. (1992) introduced a model that uses the multimodality of local velocity information to compute transparency. Multimodality is suppressed at the local velocity level in our model and appears primarily only at the output level of the model. Thus, we represent transparent phenomena at a much coarser resolution.

The selection units in our network also detected discontinuities in the distribution of motion-energy inputs, but because they were optimized to respond primarily to the patterns of discontinuities that characterize regions of reliable support for object motion, the algorithm that they implement was more selective. Thus, not all discontinuous patterns of velocity activated the selection units equally well (Fig. 5). The model selected disjoint subsets of spatial locations over which to integrate the local velocity field. This allowed the model to account for interpenetrating motion fields for which the assumption of spatial continuity of the velocity field was invalid.

Darrell and Pentland (1991) suggested a similar approach in which multiple hypotheses competed to include samples within their regions of support, but since the number of objects was not known a priori, a complex relaxation scheme was proposed to compute both the optimal number of objects and velocities. Because our model had a fixed number of hypotheses for velocities, it was possible to estimate regions of support rapidly with a feedforward architecture.

\section{Selective attention}

The active selection mechanism in our model may represent a fundamental aspect of cortical processing that occurs in many preattentive perceptual phenomena (Treisman, 1988; Bergen and Julesz, 1983). The same mechanisms that were used to implement the covert, preattentive form of selection in our model could also be tapped to provide overt attentional processing. Attentional modulation of responses has not been reported in area MT, but other motion areas such as area MST may be better candidates (Andersen et al., 1990). Attentional bias of the selection network has the advantage of not corrupting the specific information being processed by the local velocity network, especially at threshold levels of processing. Suppression of irrelevant processing may be as important as the enhancement of the relevant part.

Attention experiments have been performed in area V4 using neurons that are selective for color and orientation. Moran and Desimone (1985) have observed that the response of a neuron in V4 to its preferred stimulus can be reduced if the monkey is asked to attend to a nonpreferred stimulus, but only if the nonpreferred stimulus is presented within the receptive field of the neuron. This agrees with our model of the local velocity network, insofar as the suppression would be the result of competition 
among neurons preferring different properties in the same spatial location (Koch and Ullman, 1985; Desimone et al., 1990;).

\section{Nonclassical surrounds}

The inhibitory effects of surround motion on selection units in our model were similar to inhibitory surround effects that have been observed in many MT cells (Allman et al., 1985; Tanaka, et al., 1986). The nonclassical surround effects in some MT cells were also dependent on direction and speed of the surround motion with the greatest inhibition occurring when speed and direction in the surround matched the optimal stimulus for the cell. The effect of whole-field motion on the responses of selection units in our model was derived from the competition among the selection units representing the same candidate velocity across large regions of the visual field. When evidence for a particular velocity was present in all regions of the image equally, very little support needed to be assigned to any one region. Thus, the presence of similar motion in surrounding regions tended to suppress the selection units. On the other hand, if support for the velocity was concentrated in a small region of the image, the selection units in these regions had much stronger responses.

In addition to having silent suppressive surrounds, selection units in our model also exhibited broader directional tuning and similarity of orientation and direction tuning suggesting that these units correspond to the "pattern" (Movshon et al., 1985) or Type II (Albright, 1984) cells found in monkeys. This overlap of properties leads to the prediction that at least some MT cells which show center-surround suppression should also exhibit pattern direction selectivity. This link between pattern direction selectivity and center-surround suppression could be tested in MT cells. Discovery of such a link would lend support to the biological viability of the proposed model; failure to find such a link, while not invalidating the model, would suggest that the selection pathway in the model may be implemented in an area other than MT.

Recently, Born and Tootell (1992) have used the 2-deoxyglucose technique to identify the spatial organization of two broad classes of cell in MT based on how these cells responded to whole field motion. "Band" cells tended to respond to some directions of whole field motion, while "interband" cells showed no strong response to any direction of whole field motion. More detailed physiological studies showed the interband cells to have inhibitory effects from motion in regions surrounding the classical field similar to those reported by other investigators. There were roughly equal numbers of cells in each class (45\% interband and $55 \%$ band cells). Our model makes the strong prediction that long-range intrinsic connections should occur between groups of cells with nonclassical receptive fields. There is already evidence that the long-range intrinsic connections in MT, as elsewhere in the neocortex, are somewhat patchy, and experiments are underway to test how well these patchy connections align with band and interband regions ( $R$. Born, personal communication). If the amplification mechanism discussed above were used to perform the soft-maximum normalization, then the longrange horizontal connections between pools of selection units may act through local inhibitory circuits.

\section{Segmentation}

The selection network provided a partial solution to the problem of image segmentation. Previous attempts to segregate figure from ground have implicitly assumed that objects were spatially continuous and that the first step was to find a bounding contour.
Our approach to segmentation does not make this assumption: the selection network may group information that is spatially separated by intervening objects. There is psychophysical evidence for such a coarse segmentation of motion signals (Bravo and Watamaniuk, 1994). A similar algorithm may be used in other areas of sensory cortex to assess the importance of information processing within local neighborhoods. Nonclassical surrounds have been reported in other areas of visual cortex, including oriented neurons in area $\mathrm{V} 1$ that have opposing orientation surrounds (Blakemore and Tobin, 1972; Knierim and Van Essen, 1992) and color-selective neurons in area V4 that have antagonistic nonclassical surrounds (Desimone et al., 1985).

Neurons in other areas of the visual cortex may represent different properties of the same object, which raises the issue of how motion segregation in area MT could be used to improve processing of visual and nonvisual stimulus properties in other areas of the brain. Area MT projects subcortically and could be used to direct eye movements to interesting regions of an object. Integration could also be achieved by feedback projections to area V1 and V2 (Finkel and Sajda, 1993); in this way, partial information about motion segmentation in area MT could be used to help organize the preprocessing for other cortical areas, such as V2 and V4, which form the ventral pathway into the inferior temporal cortex. Conversely, information about form and color in the ventral areas could, by feedback projections back to area V1, be used to help segregated moving stimuli (Stoner and Albright, 1993). We suggest that the mixing of information between cues and modalities by feedback projections occurs primarily through the selection units.

\section{Gain normalization of selection units}

The selection process has two phases. First, the selection network at each spatial location rapidly computes a selection value for each broadly tuned velocity unit. This feedforward operation is followed by soft-maximum normalization across spatial locations for each vclocity, converting the relative value to an absolute probability. The response normalization can be implemented by a network with inhibitory interactions (Heeger, 1992) and similar mechanisms have been proposed for other attentional phenomena (Hadeler, 1974; Sandon, 1990). However, reciprocal inhibition is relatively slow to converge and cortical circuits may take advantage of recurrent excitation within networks of cortical pyramidal neurons to speed up the normalization (Douglas et al., 1989). Instead of direct inhibition, the units representing a particular direction of motion within a column could reduce the amplification of units with similar direction preferences in other columns. This is similar to using presynaptic inhibition to implement a winner-take-all (Yuille and Grzywacz, 1989).

The gain of local cortical amplification, which is under inhibitory control (R. Douglas and M. Mahowald, personal communication), is equivalent to the gain of soft-maximum, the value of $\alpha$ required in Eq. 1 . It may be useful to dynamically adapt the local amplification factor according to the properties of incoming stimuli and also through feedback from higher levels of processing. One danger with this approach is that too high a gain could result in instabilities within the positive feedback loop, resulting in phase-locked oscillations and insensitivity to inputs. Synchronization of the spike firing among a pool of cells might, however, serve to enhance their impact on mutual postsynaptic targets. This suggests that some of the rhythms that have been observed in brain circuits, both in aroused and sleep 
states, might be the endpoint of selection operations (Steriade et al., 1993).

The function of the intrinsic collaterals predicted by the model is to compare the relative activity levels within different columns and to regulate local mechanisms that gate the flow of information to other areas of the brain. The physiological effects of these collaterals should be the suppression of activity if activity in neighboring columns is high and enhancement if neighboring activity is low. However, the enhancement should only take place if there is significant local activity from feedforward inputs. Some of the layer $2 / 3$ pyramidal neurons have extensive longrange horizontal axonal arborizations that could function as long-range gain normalization and in vitro physiological studics within layer $2 / 3$ have provided evidence for both suppressive and enhancing effects (Hirsch and Gilbert, 1991). This suggests that selection cells may be concentrated within the upper layers of cerebral cortex. Since the output neurons to subcortical areas are in the deeper layers, selection neurons in the upper layers might control the output from the deeper layers. If the corresponding local velocity neurons, selection neurons, and output neurons were in different layers of the same column, the lengths of axons needed to connect them would be minimized.

The rate of normalization is limited by the speed with which information can be transmitted horizontally within the cortex or by feedback loops between areas. In our simulations of transparent and occluding visual stimuli, the responses of the selection units varied more rapidly than the output units as moving objects traversed their receptive fields. An output received converging subunits corresponding to the local velocity inputs, which were rapidly gated by the selection units as the most reliable regions moved across the visual field. The selected subunits would vary from moment to moment and be dynamically reorganized during motion processing even though the response of the output unit would remain stable. Such subunits might not be apparent for simple stimuli but could be probed with motion stimuli containing competing velocities. These motion stimuli could be used to distinguish selection units from output units in single-unit recordings. Van Essen and Anderson (1990) have also proposed control circuits for 'shifting" subunits in visual cortex, but their purpose was to spatially translate the inputs rather than to select information.

\section{Extensions of the model}

The present model does not include mechanisms for the temporal integration of motion in the computation of velocity, as used in the human visual system (McKee and Welch, 1985). There are also a number of non-Fourier motion stimuli that require some form of temporal integration to detect the motion in these stimuli (Albright, 1992; Victor and Conte, 1992; Wilson et al., 1992). Experiments by Watamaniuk and McKee (1993) also show that human subjects can detect the motion of a single dot in the midst of a random-dot cinematogram, if the dot has a fixed repeatable trajectory. It should be possible to extend the current model to account for thesc results by including mechanisms for temporal integration.

The present model was designed for rigid objects translating uniformly. A system for detecting more complex motions would require a more sophisticated output layer. The single output column in the model could be replicated in a retinotopically organized array that could serve as the input for processing visual stimuli with nonuniform flow fields, such as expansion and rotation. Neurons with these properties have been found in area MST, which receives a direct projection from area MT (Saito et al., 1986; Duffy and Wurtz, 1991). Nonrigid motions in three dimensions, such as those used by Nakayama and Silverman (1988), could by interpreted be this extended version of the model.

MT neurons are known to selectively respond to binocular disparity as well as velocity, suggesting that they may be representing the three-dimensional velocities of objects. Selection networks analogous to the ones introduced here for motion could be used in binocular vision to assess the reliability of stereoscopic correspondences and also to group nearby regions of the visual field belonging to the same object even when there are transparcnt surfaces. Finally, the partial segmentation of motion provided by our model could be integrated into a more global scheme for object recognition.

\section{Conclusions}

We have introduced a novel strategy for motion processing that depends on explicitly representing the reliability of local velocity estimates by special selection units. Although the selection units are partially velocity tuned, it is clear in the model that they do not represent the local velocity but, instead, decide which local velocity estimates should be included in further processing. Therefore, the fact that a neuron is velocity tuned is not sufficient evidence to conclude that its function must be to represent local velocity. Some neurons in the visual cortex, like the selection units, may be more concerned with selecting and grouping information than with representing the information itself. The separation of control from representation is essential to the selection process that governs the performance of our model.

One of the reasons the model deals well with occlusion and transparency, and can compute reliable velocity estimates rapidly, is because it is does not attempt to construct a motion flow field throughout space or for all parts of an object, as in many previous models of motion (Marr, 1982). In contrast, we only represent explicitly those selected parts that are particularly salient and unambiguous. This can greatly simplify the integration and interpretation of motion signals, but it means that our model does not attempt to solve the full object segmentation problem. While selected regions that share the same local velocity can be easily grouped based on signals from the model, these regions may not represent all of an object (Fig. 6) or they may correspond to more than a single object. Some further processing would be required to complete object segmentation.

The type of partial segmentation cues provided by the sclection pathway in the model suggests a strategy for object segmentation based on combining partial segmentation information from many different perceptual features, such as color, texture and motion. Selected regions that share a common value of a particular feature can be grouped more easily and assigned to an object, which may be of advantage for the ventral processing stream concerned with object recognition. With fewer salient features to match, the combinatorial problem of finding the correct match is greatly simplified. Reduced representations based on reliability may also be helpful in learning relationships between representations since robustness is already a part of the representation (Ballard and Whitehead, 1990; Churchland et al., 1994).

\section{References}

Adelson EH, Bergen JR (1985) Spatiotemporal energy models for the perception of motion. J Opt Soc Am [A] 2:284-299. 
Adelson M, Movshon JA (1982) Phenomenal coherence of moving visual patterns. Nature 300:523-525.

Albrecht DG, Geisler WS (1991) Motion sensitivity and the contrastresponse function of simple cells in the visual cortex. Vis Neurosci $7: 531-546$.

Albright T (1984) Direction and orientation selectivity of neurons in visual area MT of the macaque. J Neurophysiol 52:1106-1130.

Albright TD (1992) Form-cue invariant motion processing in primate visual cortex. Science 255:1141-1143.

Allman J, Miezin F, McGuinnes E (1985) Stimulus-specific responses from beyond the classical receptive field: neurophysiological mechanisms for local-global comparisons in visual neurons. Annu Rev Neurosci 8:407-430.

Andersen RA, Siegel RM (1989) Motion processing in primate cortex. In: Signal and sense: local and global order in perceptual maps (Edelman GM, Gall WE, Cowan WM, eds). New York: Wiley.

Andersen R, Graziano M, Snowden R (1990) Translational invariance and attentional modulation of MST cells. Soc Neurosci Abstr 16:7.

Andrews BW, Pollen TA (1979) Relationship between spatial frequency selectivity and receptive field profile of simple cells. J Neurophysiol 287:163-176.

Ballard DH, Whitehead SD (1990) Active perception and reinforcement learning. Neural Comput 2:409-419.

Bergen JR, Julesz B (1983) Rapid discrimination of visual patterns. IEEE Trans Sys Man Cybern 13:857.

Blakemore C, Tobin EA (1972) Lateral inhibition between orientation detectors in the cat's visual cortex. Exp Brain Res 15:439-440.

Bonds AB (1989) Role of inhibition in the specification of orientation selectivity of cells in the cat striate cortex. Vis Neurosci 2:41-55.

Born RT, Tootell RBH (1992) Segregation of global and local motion processing in primate middle temporal visual area. Nature 357:497500.

Braddick OJ (1974) A short-range process in apparent motion. Vision Res 14:519-527.

Braddick OJ (1993) Segmentation versus integration in visual motion processing. Trends Neurosci 16:263-268.

Bravo MJ, Watamaniuk SNJ (1994) Evidence for two speed signals: a course local signal for segregation and a precise global signal for discrimination. Vision Res, in press.

Britten KH, Shadlen MN, Newsome WT, Movshon JA (1992) The analysis of visual motion: a comparison of neuronal and psychophysical performance. J Neurosci 12:4745-4765.

Carandini M, Heeger DJ (1994) Summation and division by neurons in primate visual cortex. Science 264:1333-1336.

Churchland PS, Sejnowski TJ (1993) The computational brain. Cambridge, MA: MIT Press.

Churchland PS, Ramachandran VS, Sejnowski TJ (1994) Critique of pure vision. In: Large-scale neuronal theories of the brain (Koch C, Davis J, eds). Cambridge, MA: MIT Press.

Darrell T, Pentland A (1991) Robust estimation of a multi-layered motion representation. In: Proceedings IEEE workshop on visual motion. Princeton, NJ: IEEE.

Desimone R, Schein SJ, Moran J, Ungerleider LG (1985) Contour, color and shape analysis beyond the striate cortex. Vision Res 25 : $441-452$.

Desimone R, Wessinger M, Thomas L, Schneider W (1990) Attentional control of visual perception: cortical and subcortical mechanisms. In: Cold Spring Harbor symposium on quantitative biology, Vol 55, The brain, pp 963-971. Cold Spring Harbor, NY: Cold Spring Harbor Laboratory.

Dobkins KR, Albright TD (1993) What happens if it changes color when it moves?: psychophysical experiments on the nature of chromatic input to motion detectors. Vision Res 33:1019-1036.

Douglas RJ, Martin KAC, Whitteridge D (1989) A canonical microcircuit for neocortex. Neural Comput 1:480-488.

Duffy CJ, Wurtz RH (1991) Sensitivity of MST neurons to optic flow stimuli. II. Mechanisms of response selectivity revealed by small-field stimuli. J Neurophysiol 65:1346-1359.

Emerson RC, Citron MC, Vaughn WJ, Klein SA (1987) Nonlinear directionally selective subunits in complex cells of cat striate cortex. J Neurophysiol 58:33-65.

Emerson RC, Bergen JR, Adelson EH (1992) Directionally selective complex cells and the computation of motion energy in cat visual cortex. Vision Res 32:203-218.

Feldman J, Ballard D (1982) Connectionist models and their properties. Cognit Sci 6:205-254.
Ferrera VP, Maunsell JHR (1991) Responses of single units in macaque VI to moving patterns. Soc Neurosci Abstr 17:177.

Finkel LH, Sajda P (1993) Object discrimination based on depthfrom-occlusion. Neural Comput 4:901-921.

Gattass R, Gross CG (1981) Visual topography of striate projection zone (MT) in the posterior superior temporal sulcus of the macaque. J Neurophysiol 46:621-638.

Grzywacz NM, Yuille AL (1990) A model for the estimation of local image velocity by cells in the visual cortex. Proc R Soc Lond [Biol] 239:129-161.

Hadeler KP (1974) On the theory of lateral inhibition. Kybernetic 14: 161-165.

Heeger DJ (1987) Model for the extraction of image flow. J Opt Soc Am [A] 4:1455-1471.

Heeger DJ (1992) Normalization of cell responses in cat striate cortex. Vis Neurosci 9:181-197.

Hildreth EC (1984) The measurement of visual motion. Cambridge, MA: MIT Press.

Hochstein S, Shapley RM (1976) Quantitative analysis of retinal ganglion cell classifications. J Physiol (Lond) 262:237-264.

Holub RA, Morton-Gibson M (1981) Response of visual cortical neurons of the cat to moving sinusoidal gratings: response-contrast functions and spatiotemporal integration. J Neurophysiol 46:1244-1259.

Horn BKP, Schunk BG (1981) Determining optical flow. Artif Intell $17: 185-203$

Jacobs RA, Jordan MI, Nowlan SJ, Hinton GE (1991) Adaptive mixtures of local experts. Neural Comput 3:79-87.

Jasinchi R, Rosenfeld A, Sumi K (1992) Perceptual motion transparency: the role of geometrical information. J Opt Soc Am [A] 9:18651879.

Kienker PK, Sejnowski TJ, Hinton GE, Schumacher LE (1986) Separating figure from ground with a parallel network. Perception 15: 197-216.

Knierim JJ, van Essen DC (1992) Neuronal responses to static texture patterns in area V1 of the alert macaque monkey. J Neurophysiol 67: 961-980.

Koch C, Ullman S (1985) Shifts in selective attention: towards the underlying neural circuitry. Hum Neurobiol 4:219.

Koch C, Wang HT, Mathur B (1989) Computing motion in the primate's visual system. J Exp Biol 146:115-139.

Levinson E, Sekuler R (1976) Adaptation alters perceived direction of motion. Vision Res 16:779-781.

Maffei L, Fiorentini A (1977) Spatial frequency rows in the striate visual cortex. Vision Res 17:257-264.

Marr D (1982) Vision. New York: Freeman.

Marr D, Ullman S (1981) Directional selectivity and its use in early visual processing. Proc R Soc Lond [Biol] 211:151-180.

Maunsell JHR (1988) Representation of three-dimensional visual space in the cerebral cortex. Can J Physiol Pharmacol 66:478-487.

Maunsell JHR, Newsome WT (1987) Visual processing in monkey extrastriate cortex. Annu Rev Neurosci 10:363-401.

Maunsell JHR, Van Essen DC (1983) Functional properties of neurons in the middle temporal visual area (MT) of the macaque monkey: I. Selectivity for stimulus direction, speed and orientation. J Neurophysiol 49:1127-1147.

McKee SP (1981) A local mechanism for differential velocity detection. Vision Res 21:491-500.

McKee SP, Nakayama K (1984) The detection of motion in the peripheral visual field. Vision Res 24:25-32.

McKee SP, Welch L (1985) Sequential recruitment in the discrimination of velocity. J Opt Soc Am [A] 2:243-251.

McKee SP, Silverman GH, Nakayama K (1986) Precise velocity discrimination despite random variations in temporal frequency and contrast. Vision Res 26:609-619.

McLean J, Palmer LA (1989) Contribution of linear spatiotemporal receptive field structure to velocity selectivity of simple cells in area 17 of cat. Vision Res 29:675-679.

McLean J, Palmer LA (1994) Organization of simple cell responses in the three-dimensional (3-D) frequency domain. Vis Neurosci 11: 295-306.

McLean J, Raab S, Palmer LA (1994) Contribution of linear mechanisms to the specification of local motion by simple cells in areas 17 and 18 in the cat. Vis Neurosci 11:271-294.

Mikami A, Newsome WT, Wurtz RH (1986) Motion selectivity in macaque visual cortex: II. Spatio-temporal range of directional interactions in MT and V1. J Neurophysiol 55:1328-1339. 
Moran J, Desimone R (1985) Selective attention gates visual processing in the extrastriate cortex. Science 229:782-784.

Morgan MJ, Ward R (1980) Conditions for motion flow in dynamic visual noise. Vision Res 20:431-435.

Movshon JA, Thompson ID, Tolhurst DJ (1978) Receptive field organization of complex cells in the cat's striate cortex. J Physiol (Lond) 283:79-99.

Movshon JA, Adelson EH, Gizzi MS, Newsome WT (1985) The analysis of moving visual patterns. In: Pattern recognition mechanisms (Chagas C, Gattass R, Gross C, eds), pp 117-151. New York: Springer.

Nagel HH (1987) On the estimation of optical flow: relations between different approaches and some new results. Artif Intell 33:299-324.

Nakayama K (1985) Biological image motion processing: a review. Vision Res 25:625-660.

Nakayama K, Silverman GH (1988) The aperture problem-I. Perception of nonrigidity and motion direction in translating sinusoidal lines. Vision Res 28:739-746.

Nakayama K, Tyler CW (1981) Psychophysical isolation of movement sensitivity by removal of familiar position cues. Vision Res 21:427433.

Newsome WT, Pare EB (1988) A selective impairment of motion perception following lesions of the middle temporal visual area (MT). J Neurosci 8:2201-2211.

Newsome WT, Gizzi MS, Movshon JA (1983) Spatial and temporal properties of neurons in macaque MT. Invest Ophthalmol Vis Sci 24:106.

Newsome WT, Wurtz RH, Dursteler MR, Mikami A (1985) Deficits in visual motion perception following ibotenic acid lesions of the middle temporal visual area of the macaque monkey. J Neurosci $5: 825-840$

Newsome WT, Britten KH, Movshon JA (1989) Neuronal correlates of a perceptual decision. Nature 341:52-54.

Nowlan SJ (1990) Competing experts: an experimental investigation of associative mixture models. Tech Rep CRG-TR-90-5, Department of Computer Science, University of Toronto.

Nowlan SJ, Sejnowski TJ (1993) Filter selection model for generating visual motion signals. In: Advances in neural information processing systems, Vol 5 (Hanson SJ, Cowan ID, Giles CL, eds), pp 369-376. San Mateo, CA: Kaufmann.

Nowlan SJ, Sejnowski TJ (1994) Filter selection model for motion scgmentation and velocity integration. J Opt Soc Am [A], in press.

Ohzawa I, Sclar G, Freeman RD (1985) Contrast gain control in the cat's visual system. J Neurophysiol 54:651-667.

Qian N, Andersen RA (1994) Transparent motion perception as detection of unbalanced motion signals. II. Physiology. J Neurosci, in press.

Qian N, Andersen RA, Adelson EH (1994a) Transparent motion perception as detection of unbalanced motion signals. I. Psychophysics. $\mathrm{J}$ Neurosci, in press.

Qian N, Andersen RA, Adelson EH (1994b) Transparent motion perception as detection of unbalanced motion signals. III. Modeling. J Neurosci, in press.

Ramachandran VS, Cavanagh P (1987) Motion capture anisotropy. Vision Res 27:97-106.

Robson JG (1988) Linear and nonlinear operations in the visual system. Invest Ophthalmol Vis Sci [Suppl] 29:117.

Rodman HR, Albright TD (1989) Single-unit analysis of patternmotion selective properties in the middle temporal visual area (MT). Exp Brain Res 75:53-64.
Rubin N, Hochstein S (1993) Isolating the effect of one-dimensional motion signals on the perceived direction of moving two-dimensional objects. Vision Res 33:1385-1396.

Saito H, Yukie M, Tanaka K, Hikosaka K, Fukada Y, Iwai E (1986) Integration of direction signals of image motion in the superior temporal sulcus of the macaque monkey. J Neurosci 6:145-157.

Sandon PA (1990) Simulating visual attention. J Cognit Neurosci 2:213-231

Sereno ME (1993) Neural computation of pattern motion: modeling stages of motion analysis in the primate visual cortex. Cambridge, MA: MIT Press.

Smith JA, Grzywacz NM (1993) A local model for transparent motions based on spatio-temporal filters. In: Computation and neural systems (Bower J, Eeckman F, eds). Norwell, MA: Kluwer/Academic.

Snowden RJ, Treue S, Erickson RG, Andersen RA (1991) The response of area MT and V1 neurons to transparent motion. J Neurosci $11: 2768-2785$.

Steriade M, McCormick D, Sejnowski TJ (1993) The sleeping and aroused brain: thalamocortical oscillations in neurons and networks. Science 262:629-685.

Stoner GR, Albright TD (1992) Neural correlates of perceptual motion coherence. Nature 358:412-414.

Stoner GR, Albright TD (1993) Image segmentation cues in motion processing: implications for modularity. J Cognit Neurosci 5:129149.

Stoner GR, Albright TD, Ramachandran VS (1990) Transparency and coherence in human motion perception. Nature 344:153-155.

Tanaka K, Hikosaka H, Saito H, Yukie Y, Fukada Y, Iwai E (1986) Analysis of local and wide-field movements in the superior temporal visual areas of the macaque monkey. J Neurosci 6:134-144.

Tolhurst DJ, Movshon JA (1975) Spatial and temporal contrast sensitivity of striate cortical neurons. Nature 257:674-675.

Treisman A (1988) Features and objects: the fourteenth Bartlett memorial lecture. Q J Psychol [a] 40:201-237.

Van Essen DC, Anderson CH (1990) Information processing strategies and pathways in the primate retina and visual cortex. In: An introduction to neural and electronic networks (Zornetzer SF, Davis JL, Lau C, eds), pp 43-72. San Diego: Academic.

Victor JD, Conte MM (1992) Coherence and transparency of mocing plaids composed of Fourier and non-Fourier gratings. Percept Psychophys 52:403-141.

Watamaniuk SNJ, McKee SP (1993) Why is a trajectory more detectable in noise than correlated signal dots? Invest Ophthalmol Vis Sci 34:1364.

Watson AB, Ahumada AJ (1985) Model of human visual-motion sensing. J Opt Soc Am [A] 2:322-342.

Welch $L$ (1989) The perception of moving plaids reveals two motionprocessing stages. Nature 337:734-736.

Williams DW, Sekuler R (1984) Coherent global motion percepts from stochastic local motions. Vision Res 24:55-62.

Wilson HR, Ferrera VP, Yo C (1992) A psychophysically motivated model for two-dimensional motion perception. Vis Neurosci 9:7997

Yuille AL, Grzywacz NM (1989) A winner-take-all mechanism based on presynaptic inhibition feedback. Neural Comput 1:334-347.

Zucker SW, Leclerc YG, Mohammed JL (1981) Continuous relaxation and local maximum selection: conditions for equivalence. Pattern Anal Machine Intell 3:117-128. 